\title{
Frequency and Intensity Response Properties of Single Neurons in the Auditory Cortex of the Behaving Macaque Monkey
}

\author{
GREGG H. RECANZONE, ${ }^{1,2}$ DARREN C. GUARD, ${ }^{1}$ AND MIMI L. PHAN ${ }^{1}$ \\ ${ }^{1}$ Center for Neuroscience and ${ }^{2}$ Section of Neurobiology, Physiology and Behavior, University of California, Davis, \\ California 95616
}

\begin{abstract}
Recanzone, Gregg H., Darren C. Guard, and Mimi L. Phan. Frequency and intensity response properties of single neurons in the auditory cortex of the behaving macaque monkey. J. Neurophysiol. 83: 2315-2331, 2000. Response properties of auditory cortical neurons measured in anesthetized preparations have provided important information on the physiological differences between neurons in different auditory cortical areas. Studies in the awake animal, however, have been much less common, and the physiological differences noted may reflect differences in the influence of anesthetics on neurons in different cortical areas. Because the behaving monkey is gaining popularity as an animal model in studies exploring auditory cortical function, it has become critical to physiologically define the response properties of auditory cortical neurons in this preparation. This study documents the response properties of single cortical neurons in the primary and surrounding auditory cortical fields in monkeys performing an auditory discrimination task. We found that neurons with the shortest latencies were located in the primary auditory cortex (AI). Neurons in the rostral field had the longest latencies and the narrowest intensity and frequency tuning, neurons in the caudomedial field had the broadest frequency tuning, and neurons in the lateral field had the most monotonic rate/level functions of the four cortical areas studied. These trends were revealed by comparing response properties across the population of studied neurons, but there was considerable variability between neurons for each response parameter other than characteristic frequency $(\mathrm{CF})$ in each cortical area. Although the neuronal CFs showed a systematic spatial organization across AI, no such systematic organization was apparent for any other response property in AI or the adjacent cortical areas. The results of this study indicate that there are physiological differences between auditory cortical fields in the behaving monkey consistent with previous studies in the anesthetized animal and provide insights into the functional role of these cortical areas in processing acoustic information.
\end{abstract}

\section{IN T R O D U C T I O N}

Studies in the macaque monkey auditory cortex have defined several cortical fields based on physiological, anatomic, and combined techniques (Cipolloni and Pandya 1991; Hackett et al. 1998a,b; Jones et al. 1995; Kosaki et al. 1997; Merzenich and Brugge 1973; Molinari et al. 1995; Morel et al. 1993; Pandya and Rosene 1993; Rauschecker et al. 1995, 1997). These experiments have led to a view of the primate auditory cortex wherein a "core" region is made up of the primary (AI) and rostral (R) fields surrounded by a "belt" region composed of the caudomedial field (CM) and two or more lateral fields (see Jones et al. 1995; Kaas et al. 1999; Rauschecker 1998). A

The costs of publication of this article were defrayed in part by the payment of page charges. The article must therefore be hereby marked "advertisement" in accordance with 18 U.S.C. Section 1734 solely to indicate this fact. second series of cortical fields are located more laterally and comprise the parabelt region (see Hackett et al. 1998a,b).

Although there has been a renewed interest in macaque auditory cortical anatomy and physiology, very few studies have investigated the response properties of these neurons in behaving monkeys (e.g., Ahissar et al. 1992; Benson and Hienz 1978; Benson et al. 1981; Pfingst and O'Connor 1981; Pfingst et al. 1977; Ryan et al. 1984). The effects of anesthesia and attention appear to play crucial roles in the responses of auditory neurons (Hubel et al. 1959), particularly in primates (Benson et al. 1981; Miller et al. 1972; Ryan et al. 1984; see Miller et al. 1980), which underscores the importance of quantifying the response properties of these neurons in the behaving animal. Combined physiological and anatomic studies commonly employ the definition of auditory cortical fields based on characteristics of the frequency and intensity tuning profiles to brief tonal stimuli (e.g., Kosaki et al. 1997; Morel et al. 1993; Rauschecker et al. 1997), but how these responses in the anesthetized monkey relate to those observed in the behaving animal have yet to be rigorously investigated.

Several studies in the anesthetized cat (see Schreiner 1998 for review) and owl monkey (Recanzone et al. 1999) have indicated that the response latency as well as several frequency and intensity tuning parameters are systematically organized within AI. The similarity in the functional organization of AI in different species suggests that it may be a common property of auditory cortex, similar to the functional organization of other cortical areas (see Felleman and Van Essen 1991). If this is in fact the case, a similar type of organization should be apparent in the awake macaque monkey.

In the present study, we have quantitatively defined the response properties of single neurons in the primary and surrounding auditory cortical areas in macaque monkeys performing an auditory discrimination task. We found that the basic functional properties of auditory cortical neurons were consistent with those reported previously in the anesthetized monkey and describe quantitative differences in the threshold, peak activity, latency, frequency tuning, and intensity tuning parameters between neurons in the primary and surrounding auditory cortical fields. We also noted considerable variability in these response properties across neurons and failed to reveal a systematic representation within any cortical field for any response property other than characteristic frequency.

\section{METHODS}

All experiments were performed on two adult male rhesus monkeys (Macaca mulatta) weighing 7-11 kg over the course of the study. All 
procedures followed the National Institutes of Health policies for the care and use of experimental animals and were approved by the institute animal care and use committee.

\section{Stimuli and apparatus}

Experiments were conducted in a double-walled sound attenuating booth measuring $2.4 \times 3.0 \times 2.0 \mathrm{~m}(1 \times \mathrm{w} \times \mathrm{h}$; inner dimensions $)$ lined with echo-attenuating foam (Sonex). The monkey sat in an acoustically transparent primate chair and performed a go/no-go sound localization task. The monkey was required to depress a lever to initiate each trial. Three to eight tone stimuli (750-ms interstimulus interval) then were presented from a speaker located at a distance of $1.4 \mathrm{~m}$ directly opposite the contralateral ear. A stimulus then was presented from a location in front of the monkey within $\pm 30^{\circ}$ in azimuth and/or elevation, and the monkey was required to release the lever to obtain a fluid reward. This behavioral task therefore required the monkey to attend to the location of the stimulus but not to the frequency or intensity of the stimulus.

Stimuli were generated using TDT hardware and software and all stimulus delivery and data collection were controlled by a PC. Tone bursts (50-ms duration, 3-ms linear rise/fall) were presented from the speaker located directly opposite to the contralateral ear. For each neuron, 31 different frequencies (2-5 octaves equally spaced on a logarithmic scale) were presented at 16 different intensities, generally spanning a range of $80 \mathrm{~dB}$, and each stimulus frequency and intensity was presented two times in an experimental session $(2 \times 496=992$ total stimuli). Stimuli were presented in pseudorandom order such that each stimulus was at least three frequency and intensity steps different from the preceding stimulus. Stimulus intensity at the center of the monkey's interaural axis was found to be relatively constant over a frequency range of $1-12 \mathrm{kHz}$, with a fall-off of $\sim 6 \mathrm{~dB}$ /octave at lower and higher intensities. All intensities were transformed to dB SPL off-line using this speaker transformation function.

\section{Data collection}

Monkeys were fitted with a restraining head post and recording cylinder under aseptic conditions allowing a vertical approach to the superior surface of the superior temporal gyrus (Pfingst and O'Connor 1980). Monkey $L$ underwent standard MRI imaging before the surgical implantation (1.5-T magnet, 3-mm slices in the frontal plane). Monkey $M$ underwent surgery to implant a second recording cylinder $\sim 18$ mo after implantation of the first cylinder.

A recording grid was placed in the cylinder (Crist et al. 1988), and single neurons were recorded by an electrode (1-3 M 2 ; FHC) inserted through a guide tube placed within the recording grid. Neuronal activity was amplified and displayed on an oscilloscope and audio speaker using conventional methods. Search stimuli consisted of tones, noise, band-passed noise, and clicks presented from either the speaker directly opposite the contralateral ear or from a frontal location. Single neuron activity was either isolated on-line electronically (Bak DIS-2) or neuronal waveforms were stored, and single neurons were sorted off-line based on spike maxima, minima, amplitude, width, and/or slope using interactive computer software. The response characteristics of these neurons first were determined audiovisually to provide a rough estimate of the tuning properties [characteristic frequency (CF) and threshold]. The frequency and intensity range for that experiment then was set for each neuron to measure as much of the response area as possible. The experiment then was run under computer control and the time of each spike was recorded either for $100 \mathrm{~ms}$ from stimulus onset, or $150 \mathrm{~ms}$ starting $50 \mathrm{~ms}$ before stimulus onset.

\section{Data analysis}

Frequency response areas (FRAs) were constructed for each neuron. For stimuli at the extremes of the tested frequency and intensity range (e.g., the highest intensities tested), the response to that stimulus was averaged between the two presentations. For other stimuli, the response was calculated using a weighted average based on the response to that stimulus and the eight stimuli at an adjacent frequency and/or intensity. The averaged response to that stimulus was assigned a weight of 1.0 , and the averaged response to each of the other eight stimuli was assigned a weight of 0.25 . For illustrations these weighted average responses were normalized to the peak response $(100 \%)$. Iso-response contours were drawn between points at 75,50 , and $25 \%$ of the peak response and demarcating the frequency and intensity stimuli that elicited responses greater than the mean plus two standard deviations of the spontaneous activity. The spontaneous rate was either calculated during the 50 -ms prestimulus period or as the firing rate during the first $3 \mathrm{~ms}$ after the stimulus onset averaged across all stimulus presentations (992 stimuli).

All other analysis was performed by computer algorithms. Driven responses were defined as the average of the two presentations of each stimulus that was greater that the mean plus 2 SDs of the spontaneous activity. CF was defined as the frequency that produced a driven response at the lowest intensity. If two or more adjacent stimulus frequencies produced driven responses at the lowest intensity, the CF was defined as the weighted mean of those responses using the formula

$$
\left(\sum F_{\mathrm{i}}^{*} N R_{\mathrm{i}}\right) /\left(\sum N R_{\mathrm{i}}\right)
$$

where $F_{\mathrm{i}}$ is each frequency that elicited a driven response at the lowest intensity and $N R_{\mathrm{i}}$ is the response at that frequency normalized by the greatest response to each of the frequencies from 1 to $n$. For example, if 1 spike was recorded at $1,000 \mathrm{~Hz}, 3$ spikes at $1,200 \mathrm{~Hz}$, and 2 spikes at $1,400 \mathrm{~Hz}$ at the lowest intensity tested, the CF was defined as $[(1000 * 0.33)+(1200 * 1.0)+(1400 * 0.67)] /(0.33+1.0+$ $0.67)=1233.3 \mathrm{~Hz}$. If the neuron had driven responses to two or more nonadjacent tested frequencies at the lowest intensity, the $\mathrm{CF}$ was defined as the frequency that elicited the greatest of those responses.

Threshold was defined as the lowest intensity that elicited a driven response at $\mathrm{CF}$. This measure was restricted to those neurons in which at least one intensity lower than threshold was tested. The latency measures were based in the averaged driven response in 2-ms time bins to the stimuli with the five frequencies closest to the CF of the neuron at each intensity ( 2 higher, 2 lower, and 1 at CF; 160 trials). The minimum latency was defined as the first time in which there was a driven response for at least three consecutive 2-ms time bins. The peak latency was defined as the time of occurrence of the greatest driven response. The intensity tuning measures were based on the averaged driven responses to the five stimuli with frequencies closest to the CF for each intensity (10 trials/intensity). The firing rate index was calculated as the ratio of the driven response at the highest intensity tested divided by the greatest driven response at any tested intensity. This ratio varies from 1.0 (the greatest response was at the highest intensity) to 0 (no response at the highest intensity). The intensity index was defined as the intensity of the stimulus that elicited the greatest driven response divided by the highest intensity tested. This value can range from 1.0 (the greatest response was at the highest intensity) to $\sim 0.0125$ (greatest response at an intensity $80 \mathrm{~dB}$ less than the highest intensity). The best intensity was defined as the intensity that elicited the greatest driven response.

Frequency tuning was defined by calculating the low- and highfrequency edges of the driven responses at 10 and $40 \mathrm{~dB}$ above threshold. The low-frequency border was defined as the lowest frequency that a stimulus produced a driven response before stimuli with two successively lower frequencies did not produce a driven response. The high-frequency border was defined in a similar manner for the stimuli with frequencies greater than the CF. Bandwidth was measured as the difference between the base 2 logarithm of high- and low-frequency borders. $Q$ values were defined as the difference between the high- and low-frequency borders divided by the CF. 
Linear regression analysis using two dependent variables (Mosteller et al. 1983) and second-order regression analysis determined that there was not a significant correlation between $\mathrm{CF}$ and the peak activity, firing rate index, intensity index, and best intensity. Comparisons between these response parameters as a function of cortical area was performed using a Kolmogorov-Smirnov test for each pair (6 total). To accommodate multiple tests, $P$ values of 0.01 were taken as statistically significant. For all other response parameters, there was a relationship with the $\mathrm{CF}$, and therefore Monte Carlo analysis was performed. Measured values were parsed into $1 / 2$ octave bins as a function of CF. The total signed error in each cortical area was calculated as the sum of the differences between the measured value for each neuron and the average of all AI neurons with CFs within that $1 / 2$ octave frequency range. A computer program then randomly assigned a cortical area for each value within each bin with the number of points for each cortical area the same as that measured experimentally. The total signed error was calculated as above for each of 1,000 iterations of this simulation. The error measured experimentally then was compared with the distributions of errors generated from these iterations. If the simulations created errors as great as or greater than the total error measured experimentally in $<50$ iterations the difference was considered statistically significant (50/ $1000=0.05$ ).

The physiological border between AI and R was ambiguous (see following text), and therefore all analysis was repeated for R and AI neurons in which recording locations that were used to define the border were omitted. This resulted in 36 neurons in $\mathrm{R}$ that were used in these comparisons. In all cases, the statistical significance of the results were not different depending on which population of $\mathrm{R}$ neurons were used, and therefore only the results when all $\mathrm{R}$ were tested are reported.

\section{RES ULTS}

This report is based on recordings of 604 single neurons from two monkeys. In monkey $L$, we quantified the responses of 393 neurons from 125 cortical locations in the left hemisphere. In monkey $M$, we quantified the responses of 178 neurons from 102 locations in the left hemisphere, and 33 neurons from 16 cortical locations in the right hemisphere (see Table 1). Sessions in which the behavioral performance was $<90 \%$ correct were not included in the quantitative analysis.

\section{Representation of $C F$}

The reconstructions of the $\mathrm{CF}$ defined using the standard orientation of the recording grid are shown for the left hemisphere of both monkeys in Fig. 1. $A$ and $B$ show the CF defined by either averaging the $\mathrm{CF}$ for all individual neurons (boldface type) or qualitatively defined at locations where a complete single neuron dataset was not collected (spacing is $1 \mathrm{~mm}$ ). For monkey $M$, we also collected data from 10 neurons at six locations after rotating the grid by $30^{\circ}$ to investigate neuronal responses at intermediate grid locations (data not shown). Figure 1, $C$ and $D$, reconstructs the CF map for these two hemispheres. These pseudocolor plots show the CFs in 1 octave bands for neurons with low CFs (dark blue) to high CFs (red). In both monkeys there was a central region with a clear progression of $\mathrm{CF}$ with the lowest frequency CFs located rostrally and the highest frequency CFs located progressively caudally with less variation in the medial-lateral direction. This $\mathrm{CF}$ progression is consistent with the isofrequency organization of AI described in anesthetized macaque monkeys (Kosaki et al. 1997; Merzenich and Brugge 1973; Morel et al. 1993; Rauschecker et al. 1997) and is demarcated by the heavy lines. In monkey $L$, this isofrequency representation was disrupted at the lateral, caudal, and medial borders, consistent with the lateral (L), and CM divisions of the auditory cortex. For monkey $M$, there was a reversal of the tonotopic organization rostral to $\mathrm{AI}$, consistent with area $\mathrm{R}$. The $\mathrm{CFs}$ of neurons located medial to AI in this monkey were also consistent with CM.

Figure 2 shows frontal sections from the MRI images that correspond to the region investigated in monkey $L$. The recording cylinder was targeted to overlie the center image (Fig. 2C) located at $+6 \mathrm{~mm}$ anterior in stereotaxic coordinates. The adjacent 3-mm-thick images were taken from the approximate anterior-posterior location shown in Fig. 2E. The gross structure of the cerebral cortex at these locations is consistent with previous anatomic studies of AI and further support the demarcation described in the preceding text (Cipolloni and Pandya 1991; Merzenich and Brugge 1973; Morel et al. 1993; Pandya and Rosene 1993).

Figure 3 shows representative FRAs from single neurons recorded from locations roughly parallel to the isofrequency contours in monkey L (Fig. 3H). Starting most laterally (Fig. 3, $A$ and $G)$, the responses showed several low intensity peaks and generally had either broad response areas or were poorly responsive to these tonal stimuli, indicated by the maximum response $(100 \%)$ shown above each plot. The frequency tuning of AI cells were relatively sharp by comparison (Fig. 3, $B-E$ ). At the most medial portion of the investigated area, the neurons typically became more broadly tuned again, consistent with this region corresponding to the caudomedial field CM. Direct comparison of these FRAs are shown in Fig. 3I, where the 25\%

TABLE 1. Numbers of neurons

\begin{tabular}{|c|c|c|c|c|c|c|}
\hline \multirow[b]{2}{*}{ Area } & \multicolumn{2}{|c|}{ Monkey M } & \multicolumn{2}{|c|}{ Monkey L } & \multicolumn{2}{|c|}{ Total } \\
\hline & No. of Loc. & No. of FRAs & No. of Loc. & No. of FRAs & No. of Loc. & No. of FRAs \\
\hline AI & 61 & 145 & 64 & 268 & 125 & 413 \\
\hline $\mathrm{L}$ & 0 & 0 & 10 & 30 & 10 & 30 \\
\hline $\mathrm{CM}$ & 18 & 23 & 35 & 95 & 53 & 118 \\
\hline $\mathrm{R}$ & 29 & 43 & 0 & 0 & 29 & 43 \\
\hline NDR & 10 & 0 & 16 & 0 & 26 & 0 \\
\hline Totals & 118 & 211 & 125 & 393 & 243 & 604 \\
\hline
\end{tabular}

Number of recording locations and number of quantified single neuron responses measured in both monkeys as a function of cortical area. Totals at the bottom

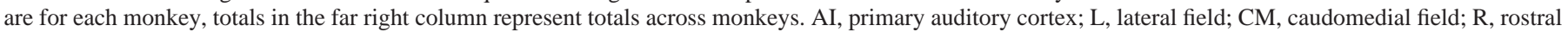
field; NDR, neurons not driven by auditory stimuli. FRA, frequency response area. 
A Recording Locations monkey $L$

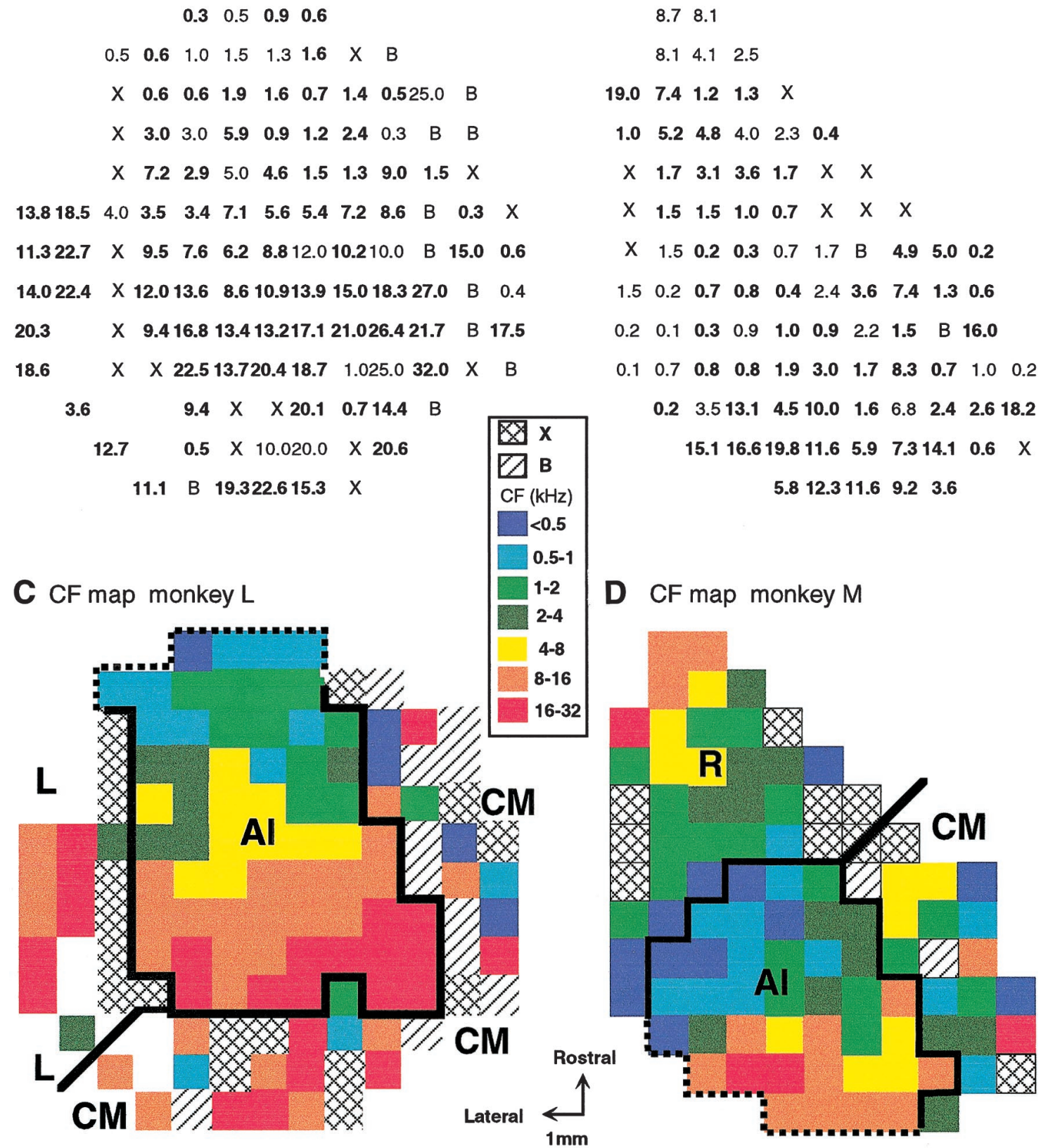

FIG. 1. Representation of characteristic frequency (CF). A: CF rounded to the nearest $0.1 \mathrm{kHz}$ measured for each recording location in monkey $L$. Bold typeface numbers indicate recording locations in which $\geq 1$ neuron was isolated and 2 complete frequency response areas (FRAs) were obtained (see METHODS). At locations where $>1$ unit was isolated, the number reflects the mean of all neurons recorded. Lighter numbers indicate locations where the responses were estimated from the multiple-unit recording by the experimenter. Locations where the $\mathrm{CF}$ could not be estimated because either the neurons responded equally to many frequencies or responded poorly to tonal stimuli but did respond to more complex stimuli are indicated by "B." Locations where no auditory driven responses were encountered are indicated by "X." $B$ : CF map from the left hemisphere of monkey $M$. Conventions as in $A$. C : pseudocolor representation of the CF map of monkey $L$. CFs were defined in 1 octave bands and assigned a different color (see inset). Locations unresponsive to acoustic stimuli are shown as cross-hatched, those where the CF could not be defined are shown as single-hatched. $D$ : pseudocolor CF map from monkey $M$. Conventions as in $C$. Heavy line is drawn to surround the region defined as primary auditory cortex (AI) and to indicate the borders with the other cortical fields (see RESULTS). Heavy dashed lines show the edges of these border regions. AI had a clear tonotopic representation that reversed at the rostral region.

contour is reproduced for the neurons shown in Fig. 3, $A-G$, on the same logarithmic frequency axis.

The lateral and caudal borders were relatively straightforward to define based on a broadening of the frequency tuning and a change in the CF. Examples of this transition are shown in Fig. 4 for monkey M. FRAs defined in AI are shown across the top row, with FRAs defined at adjacent recording locations in CM shown in the middle and bottom rows. A similar 
A

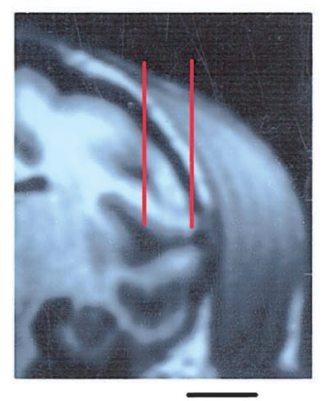

B

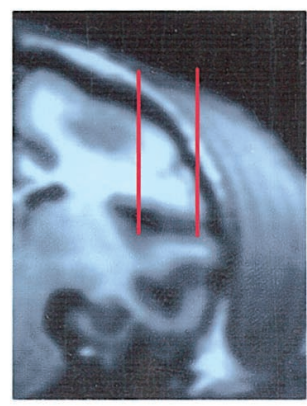

C

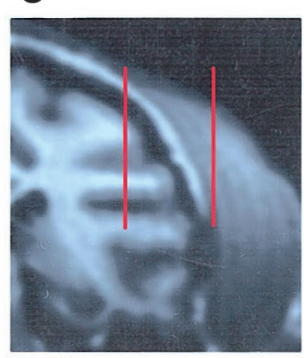

D
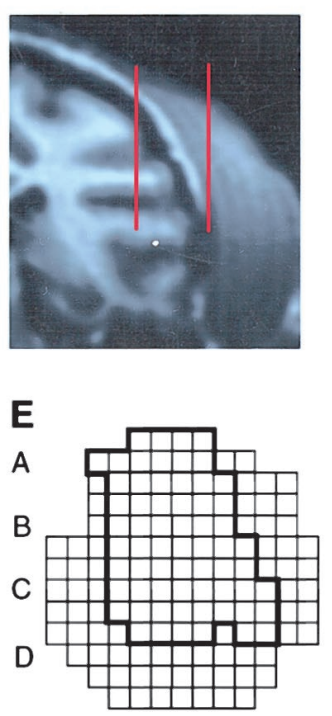

FIG. 2. Magnetic resonance (MRI) images of monkey L. MRIs (3-mm slices) in the frontal plane show the left cerebral hemisphere, mirror reversed after radiology conventions. Red vertical lines show the approximate extent of the recording locations. Panels are taken in adjacent sections and thus are $3 \mathrm{~mm}$ apart. Scale bar under $A$ is $10 \mathrm{~mm}$. Cylinder is oriented to allow a vertical approach to the superior plane of the superior temporal gyrus, which is shown at the bottom of the red vertical lines.
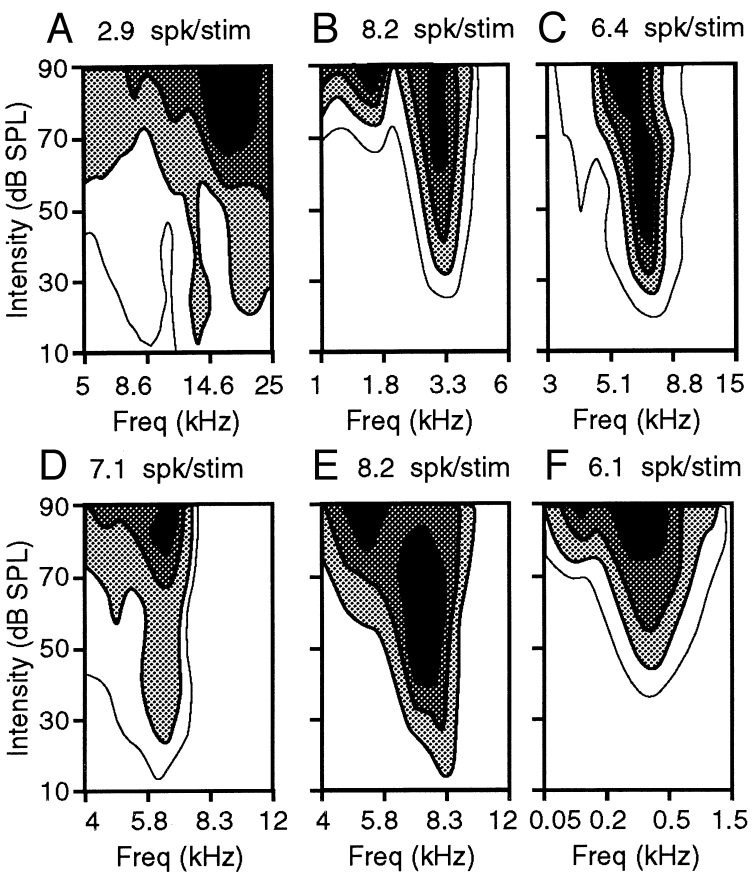

E $8.2 \mathrm{spk} / \mathrm{stim}$

F $6.1 \mathrm{spk} / \mathrm{stim}$

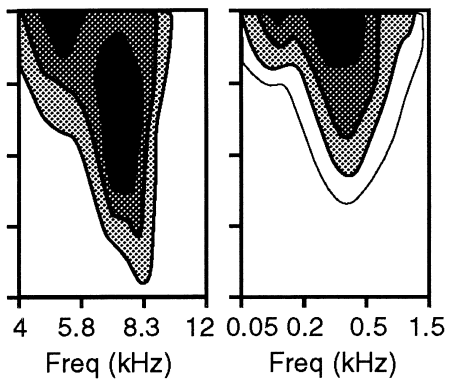

G 2.4 spk/stim

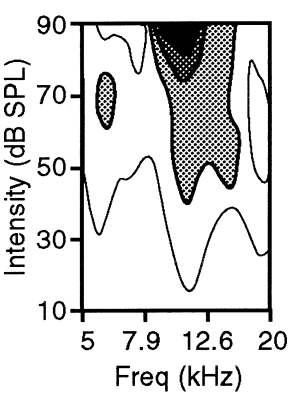

$\mathrm{H}$ Recording Locations

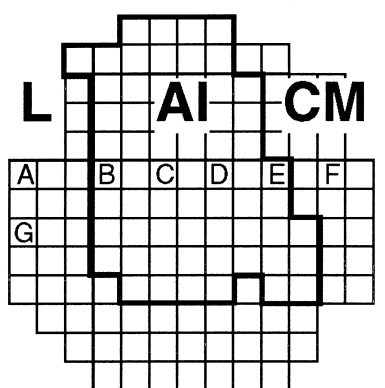

I CF and Bandwidth Comparison

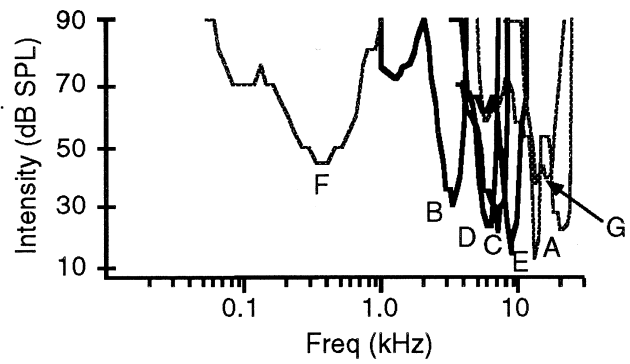

FIG. 3. Representative FRAs across the medial-lateral extent of auditory cortex. Each FRA shows the frequency and intensity responses along the $x$ and $y$ axis, respectively. Response was normalized to the peak response for each FRA (top of each plot). Black indicates stimuli that produced $>75 \%$ of the peak response, dark stippling represents 50-74\%, light stippling 25-49\%, and open regions represent $1-24 \%$ of the peak response. $A-G$ : single neuron FRAs corresponding to the location from monkey $L$ shown in $H$. $A$ and $G$ are from neurons located most laterally, $B-F$ are from neurons that were located progressively medial. Note differences in the frequency axis due to each neuron being tested with a frequency range tailored to that neuron. Frequency tuning was broadest for neurons located lateral and medial to $\mathrm{AI}(A, F$, and $G)$. $I$ reproduces the $25 \%$ response contour of each neuron on the same frequency and intensity axes. Neurons located in AI are shown with heavy solid lines $(B-E)$ and neurons in the other cortical fields are shown as thinner gray lines $(A, F$, and $G$ ). There was a clear difference in both $\mathrm{CF}$ and bandwidth for neurons outside of AI. 
A $5.2 \mathrm{spk} / \mathrm{stim}$

B $14.8 \mathrm{spk} / \mathrm{stim}$

C $1.7 \mathrm{spk} / \mathrm{stim}$
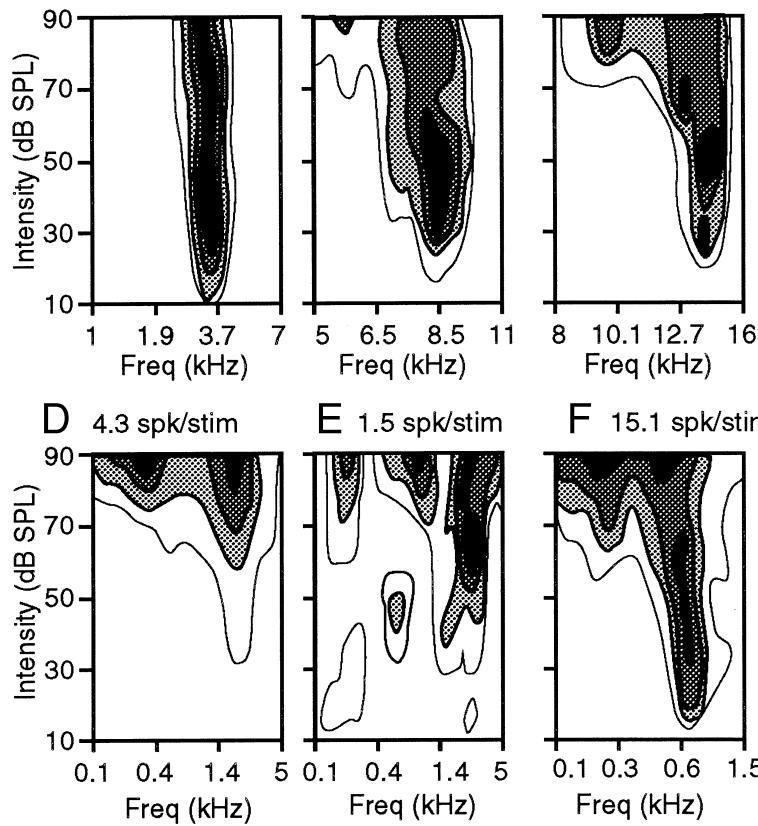

E $1.5 \mathrm{spk} / \mathrm{stim}$

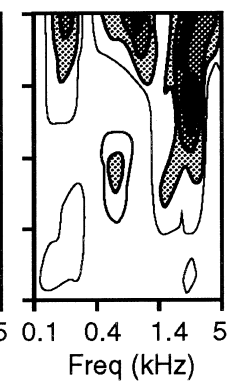

F $15.1 \mathrm{spk} / \mathrm{stim}$

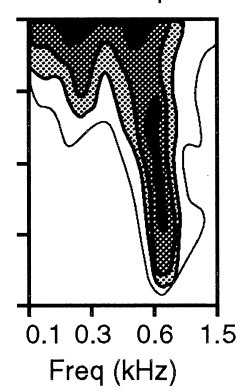

$\mathrm{H}$ Recording Locations
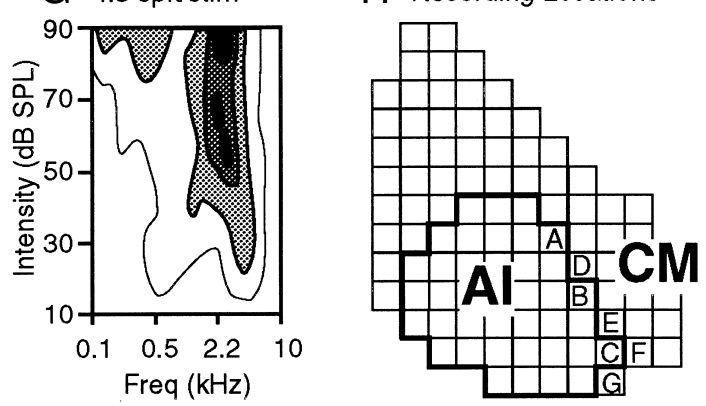

I CF and Bandwidth Comparison

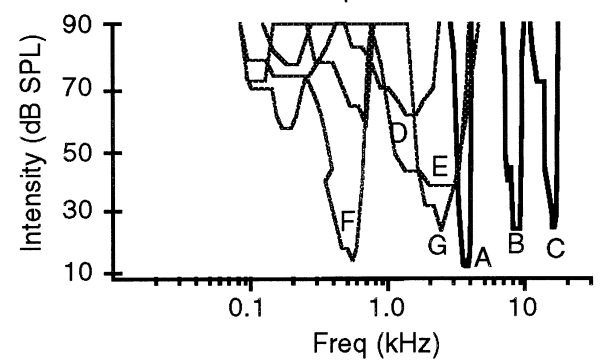

FIG. 4. Representative FRAs at the AI/caudomedial field (CM) border from monkey $M$. FRAs from single neurons are shown that straddle the AI and CM border as defined by the $\mathrm{CF}$ gradient. $A, C$, and $E$ show AI neurons. $B, D, F$, and $G$ show CM neurons, $I$ shows the $25 \%$ contour reproduced on the same frequency and intensity axes. Conventions as in Fig. 3.

example is shown for monkey $L$ in Fig. 5, with FRAs of AI neurons shown in Fig. 5, $A$ and $B$, and FRAs from neurons directly caudal to those locations and classified within $\mathrm{CM}$ shown in Fig. 5, $C$ and $D$. These two cases illustrate the characteristic change in frequency tuning, $\mathrm{CF}$, or both for neurons on either side of the AI-CM border. The AI-CM border used for the quantitative analysis described in the following text was based on this difference in both $\mathrm{CF}$ and frequency tuning bandwidth across all neurons recorded at each location. In practice, although individual FRAs could be similar between the two areas, distinct differences in the CF and frequency tuning of neurons were evident between adjacent recording locations along this border, and coupled with the response profiles assessed on-line from the multiple-unit responses, the AI-CM border was relatively straightforward to define.

The demarcation of the border between $\mathrm{AI}$ and the more rostral field was less apparent as there was not an abrupt change in $\mathrm{CF}$ across this border (Fig. 1, $B$ and $D$ ). Representative FRAs recorded in this border region are shown in Fig. 6. These neurons had similar CFs in the low-frequency region, but there did appear to be a difference in frequency bandwidth, with $\mathrm{R}$ neurons have slightly more narrow frequency tuning (see following text). Representative FRAs from neurons that were clearly rostral to AI were qualitatively similar in overall shape and threshold to those in AI (Fig. 7).

\section{Measures of activity levels}

To compare the responses of neurons between different cortical areas, each neuron was classified into one of the four

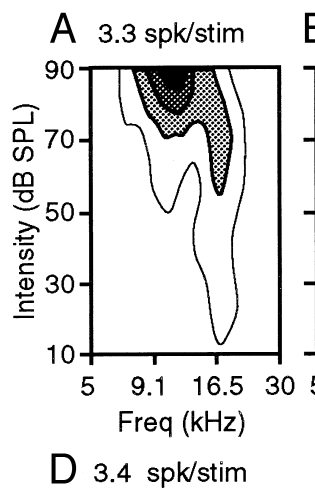

B $4.1 \mathrm{spk} / \mathrm{stim}$ C $5.3 \mathrm{spk} / \mathrm{stim}$
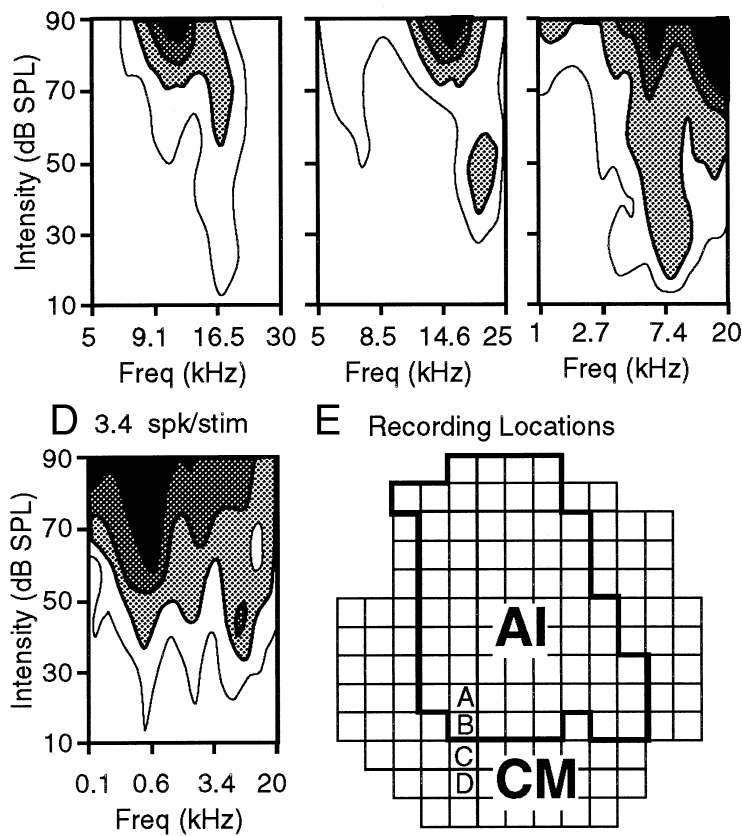

E Recording Locations

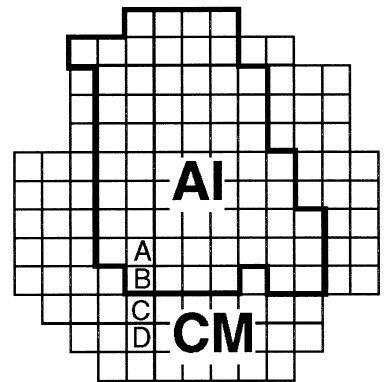

$\mathrm{F}$ CF and Bandwidth Comparison

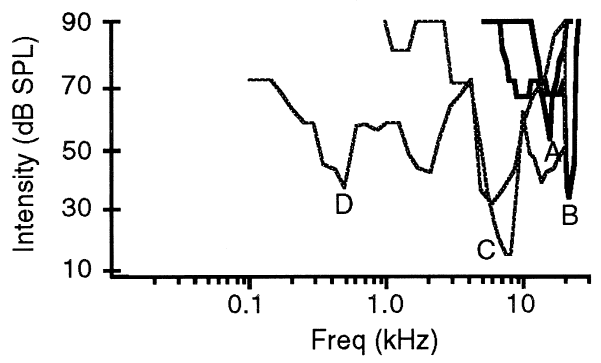

FIG. 5. Representative FRAs at the AI and CM border from monkey L. A and $B$ show single neuron FRAs at the caudal border of AI. $C$ and $D$ show single neurons at the rostral border of CM. Note the difference in $\mathrm{CF}$ and the increase in frequency tuning bandwidth between $A$ and $B$ (AI) compared with $C$ and $D(\mathrm{CM})$ shown in $F$. Conventions as in Fig. 3. FRA from the neuron shown in $B$ is drawn as continuous in $F$ for clarity. 
A $5.3 \mathrm{spk} / \mathrm{stim}$

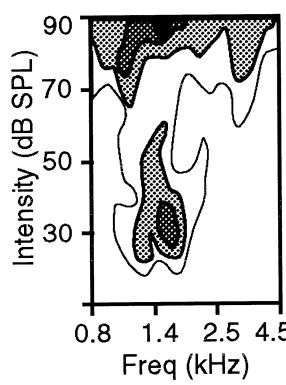

B $5.4 \mathrm{spk} / \mathrm{stim}$
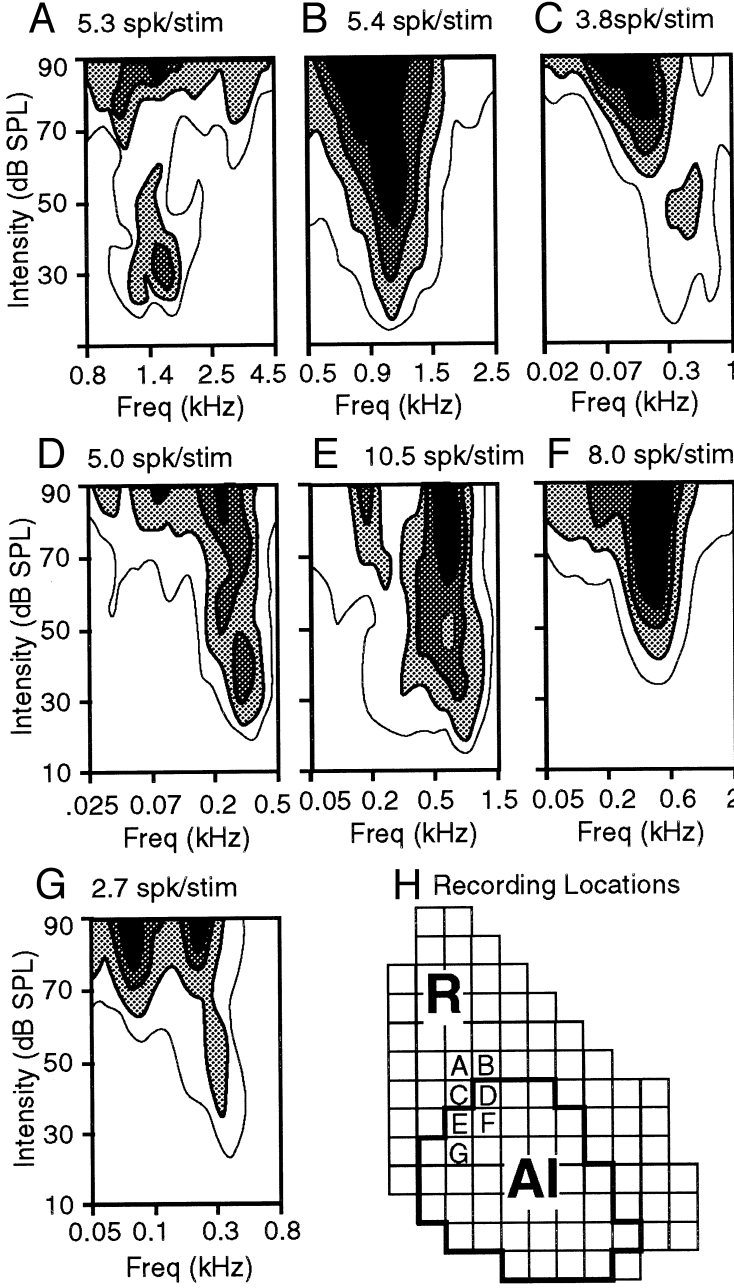

E $10.5 \mathrm{spk} / \mathrm{stim} F \quad 8.0 \mathrm{spk} / \mathrm{stim}$

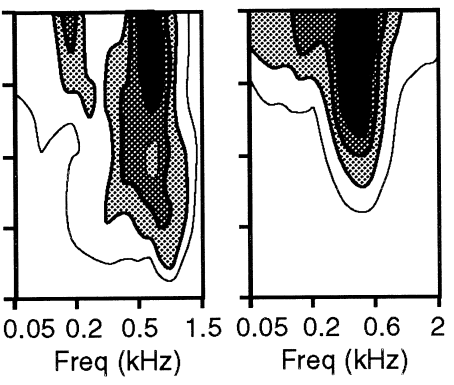

$\mathrm{H}$ Recording Locations

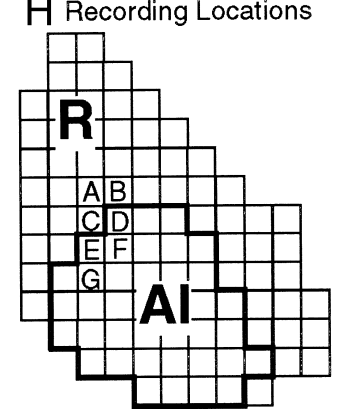

I CF and Bandwidth Comparison

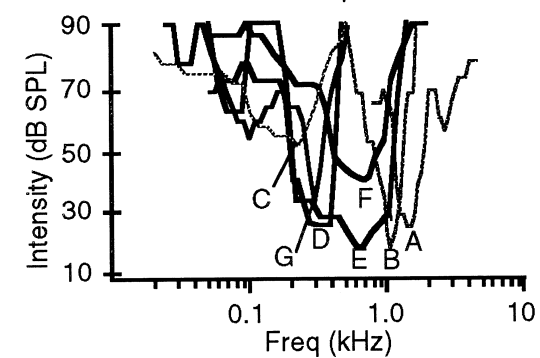

FIG. 6. Representative FRAs at the AI and rostral field (R) border in monkey $M$. A-C: FRAs from single neurons classified as being in the rostral field R. $D-G$ : FRAs from neurons classified as being within AI. There was little difference in CF or frequency bandwidth for neurons in these 2 cortical areas (I). Conventions as in Fig. 3. FRA from the neuron shown in $A$ is drawn as continuous in $I$ for clarity.

cortical areas depending on the recording locations illustrated in Fig. 1. Neurons located lateral to AI did not show clear CF reversals as described previously (Rauschecker et al. 1995), and therefore the classification of this cortical area was simplified as "L." The quantitative analysis was based on the responses relative to the $\mathrm{CF}$ of the neuron under study and did not incorporate the weighted average response for each stimulus (see METHODS).

The threshold of each cell was defined as the lowest intensity that produced a driven response. This analysis was restricted to neurons in which at least one intensity was tested below this value for all frequencies (547/604 neurons met this criterion). The thresholds for all of these AI neurons are shown in Fig. 8A. Neurons in AI with CFs near $1 \mathrm{kHz}$ had the lowest thresholds, and thresholds increased with decreasing and increasing CF, consistent with behaviorally defined audiograms in macaques (Owren et al. 1988; Stebbins et al. 1966).

Figure $8 B$ shows the mean thresholds as a function of CF for AI neurons (-) compared with those measured in each of the other three cortical areas. For this plot, neurons in each cortical area were parsed into one-half octave bins based on their CF, and each symbol shows the mean threshold for those neurons. Means and standard deviations for the thresholds and all other response parameters for all neurons in each cortical area are provided in Table 2 . Neurons in $\mathrm{CM}(\triangle)$ had significantly higher thresholds than those in AI (Monte Carlo analysis, see METHODS; $P<0.05)$. Neurons in R $(\square)$ could have higher or lower thresholds relative to neurons in AI, and these differences did not reach statistical significance, nor did the thresholds measured for neurons in L $(\bigcirc$; see Table 2). However, when only the neurons with the lowest thresholds for each one-half octave range of CFs were compared (Fig. 8C), AI neurons consistently had lower thresholds than neurons in the

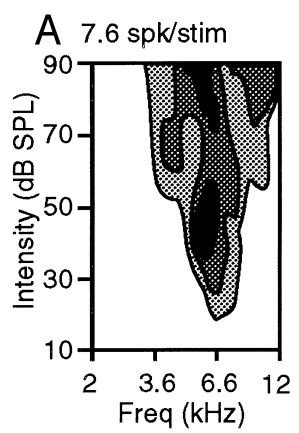

B $6.0 \mathrm{spk} / \mathrm{stim}$ C $4.2 \mathrm{spk} / \mathrm{stim}$
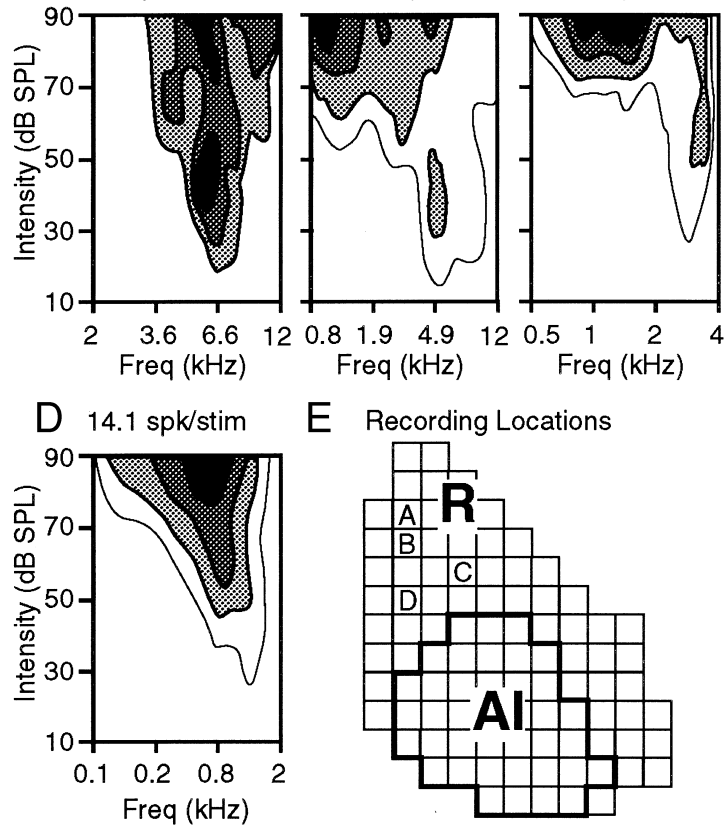

E Recording Locations

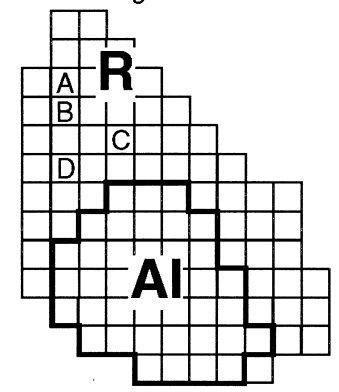

F CF and Bandwidth Comparison

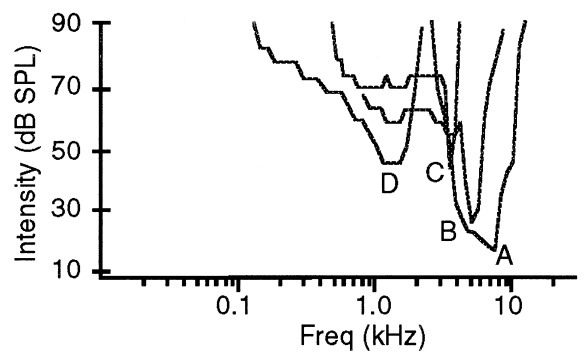

FIG. 7. Representative FRAs from the rostral field in monkey $M . A-D$ : single neuron FRAs recorded from the locations shown in $E$. These neurons were similar in many respects to those observed in AI. Conventions as in Fig. 3 . FRA from the neuron shown in $B$ is drawn as continuous in $F$ for clarity. 
A Al Neurons

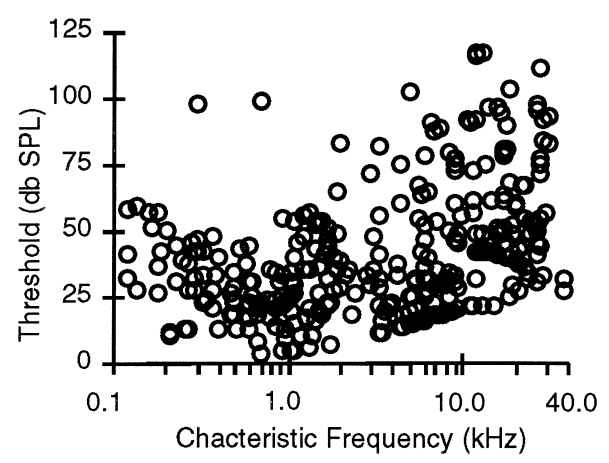

B Average Thresholds

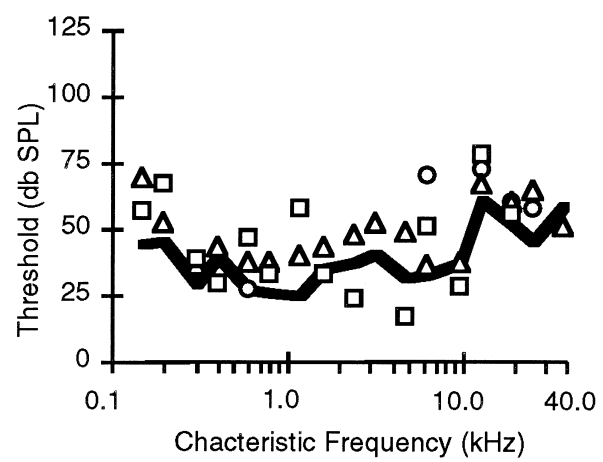

C Minimum Thresholds

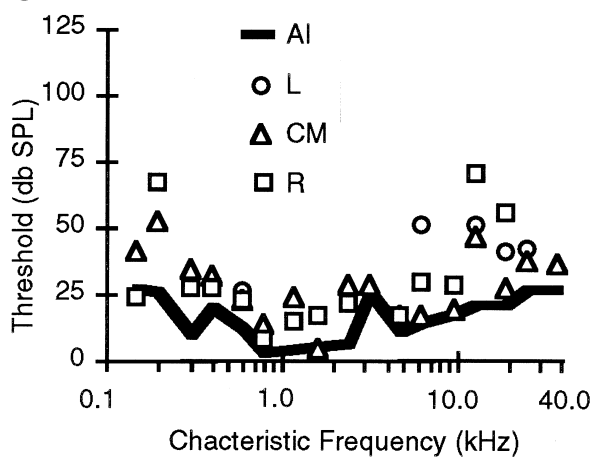

FIG. 8. Thresholds of single neurons. A: thresholds as a function of $\mathrm{CF}$ measured from all AI neurons where $\geq 1$ intensity was tested below threshold at $\mathrm{CF}(n=373)$. $B$ : mean thresholds defined for all neurons as a function of $\mathrm{CF}$. Neurons were parsed into one-half octave CF bands and plotted at the high-frequency edge of that band. - , mean threshold of AI neurons, and it therefore reflects the data from $A$. Neurons from the lateral field are shown by $\circ$, neurons from CM are shown as $\triangle$, and neurons from R are shown as $\square$. AI neurons generally had the lowest thresholds across CF compared with each of the 3 other cortical areas. $C$ : threshold of the neuron with the lowest threshold in each cortical area for each one-half octave range in CF. In this case, the AI neurons with the lowest thresholds were consistently lower than those neurons in the other cortical areas. Conventions as in $B$.

other three cortical fields, indicating that the neurons with the lowest thresholds were found in AI.

The peak activity for each neuron was defined as the highest activity measured for stimuli at the five frequencies closest to the $\mathrm{CF}$ of the neuron for each intensity tested. This measure was not significantly correlated with the $\mathrm{CF}$ of the neuron in any cortical area using linear and second-order regression $(P>$ $0.05)$, so all neurons in each area were pooled. Figure 9 shows the frequency distribution of the peak activity for neurons in each cortical area. There was no statistically significant differ- ence between neurons in $\mathrm{AI}$ and $\mathrm{CM}$ [Kolmogorov-Smirnov $(\mathrm{K}-\mathrm{S}) ; P>0.05]$. However, there was a difference between AI and $\mathrm{CM}$ and neurons recorded in the lateral field $(P<0.01)$. Neurons recorded in $\mathrm{R}$ had the lowest activity levels of the four cortical areas $(P<0.01)$.

The spontaneous activity of a sample of neurons $(43,31,11$, and 9 neurons from AI, CM, L, and R, respectively) was calculated during the $50 \mathrm{~ms}$ before stimulus onset and compared with the activity calculated from the first three milliseconds after stimulus onset, which was before neuronal activity could be stimulus driven. There was no statistically significant difference using the two measures (paired, 2-tailed $t$-test; $P>$ $0.05)$, so the 3 -ms period was used for all neurons. The mean spontaneous activity was $8.2 \pm 12.1$ spikes/s (mean $\pm \mathrm{SD}$ ), and there was no statistically significant difference between neurons either as a function of $\mathrm{CF}$ (regression analysis) or between cortical areas (K-S; $P>0.05)$.

In summary, thresholds measured to tonal stimuli in AI showed a wide range of values, but the minimum thresholds were consistent with the hearing sensitivity of macaque monkeys. CM neurons had the highest thresholds, and the neurons with the lowest thresholds were found in AI. AI and CM neurons had the highest level of activity to these tonal stimuli, with $\mathrm{R}$ neurons having the lowest level of activity (Table 2), and there was no difference in the spontaneous activity of neurons in the different cortical areas.

\section{Latency of responses}

The responses to the presentation of the five frequencies nearest CF at each intensity (160 stimuli total) were averaged in 2-ms time bins (see METHODs). The minimum latency measured in all AI neurons is shown in Fig. 10A. These latencies were widely distributed across neurons, with a range of $\sim 50$ $\mathrm{ms}$. The averaged latency as a function of $\mathrm{CF}$ for neurons in each of the four cortical areas are shown in Fig. 10B. AI and $\mathrm{CM}$ neurons had similar latencies (Monte Carlo; $P>0.05$ ). Area $\mathrm{L}$ neurons and significantly longer latencies $(P<0.05)$ and area $\mathrm{R}$ neurons had the longest latencies, which were significantly different from those measured in the three other areas $(P<0.05$; see Table 2$)$.

These differences, although statistically significant, were actually quite small due in large part to the large variance measured across neurons. Therefore the neurons with the shortest latencies in one-half octave ranges of CF were compared. This analysis revealed that the neurons with the shortest minimum latencies were found consistently in AI (Fig. 10C) with neurons in both $\mathrm{CM}$ and $\mathrm{R}$ having longer latencies. Neurons in $\mathrm{L}$ had latencies that were intermediate between these values.

The latency to the peak of the neuronal response also was compared. There was no difference in the peak latencies for neurons in the different cortical areas $(P>0.05$ for all comparisons). When the neurons with the shortest peak latency in each one-half octave range of CFs were compared, however, AI neurons had the shortest peak latencies $(P<0.05)$, neurons in $\mathrm{R}$ had the longest latencies $(P<0.05)$, and neurons in $\mathrm{CM}$ and $\mathrm{L}$ had latencies of intermediate values (data not shown).

In summary, AI neurons had the shortest minimum latencies, and $\mathrm{R}$ neurons had the longest latencies of the four cortical areas measured. The peak latency was not significantly different between neurons in the four cortical fields, although AI 
TABLE 2. Response parameters by cortical area

\begin{tabular}{|c|c|c|c|c|c|}
\hline Parameter & AI & $\mathrm{CM}$ & $\mathrm{L}$ & $\mathrm{R}$ & Statistics \\
\hline Threshold & $39.7 \pm 22.4$ & $51.5 \pm 23.1$ & $43.6 \pm 28.6$ & $43.4 \pm 28.2$ & $\mathrm{AI}=\mathrm{L}=\mathrm{R}<\mathrm{CM}$ \\
\hline Peak Act. & $39.3 \pm 33.3$ & $35.2 \pm 35.3$ & $21.5 \pm 20.2$ & $18.6 \pm 17.9$ & $\mathrm{R}<\mathrm{L}<\mathrm{CM}=\mathrm{Al}$ \\
\hline Min Lat & $32.4 \pm 13.6$ & $33.8 \pm 11.8$ & $42.8 \pm 14.3$ & $41.9 \pm 13.9$ & $\mathrm{AI}=\mathrm{CM}<\mathrm{L}<\mathrm{R}$ \\
\hline Peak Lat & $65.4 \pm 15.4$ & $64.3 \pm 12.4$ & $72.8 \pm 14.3$ & $73.1 \pm 17.3$ & $\mathrm{AI}=\mathrm{CM}=\mathrm{L}=\mathrm{R}$ \\
\hline F.R. Index & $0.83 \pm 0.18$ & $0.85 \pm 0.16$ & $0.83 \pm 0.22$ & $0.72 \pm 0.29$ & $\mathrm{R}<\mathrm{AI}=\mathrm{CM}=\mathrm{L}$ \\
\hline Int. Index & $0.77 \pm 0.21$ & $0.77 \pm 0.22$ & $0.83 \pm 0.18$ & $0.69 \pm 0.22$ & $\mathrm{R}<\mathrm{AI}=\mathrm{CM}<\mathrm{L}$ \\
\hline Best Intens. & $59.5 \pm 17.5$ & $59.6 \pm 18.6$ & $64.9 \pm 14.5$ & $49.8 \pm 16.5$ & $\mathrm{R}<\mathrm{AI}=\mathrm{CM}<\mathrm{L}$ \\
\hline BW10dB & $0.43 \pm 0.50$ & $0.60 \pm 0.56$ & $0.51 \pm 0.20$ & $0.31 \pm 0.25$ & $\mathrm{R}<\mathrm{AI}=\mathrm{L}<\mathrm{CM}$ \\
\hline BW40dB & $0.75 \pm 0.80$ & $0.92 \pm 0.97$ & $0.84 \pm 0.40$ & $0.63 \pm 0.50$ & $\mathrm{R}<\mathrm{AI}=\mathrm{L}<\mathrm{CM}$ \\
\hline Q10dB & $53.8 \pm 181.7$ & $12.6 \pm 31.5$ & $42.0 \pm 109.5$ & $180.9 \pm 372.8$ & $\mathrm{R}>\mathrm{AI}=\mathrm{L}>\mathrm{CM}$ \\
\hline Q40dB & $32.9 \pm 142.0$ & $10.1 \pm 15.7$ & $27.7 \pm 100.8$ & $84.8 \pm 277.4$ & $\mathrm{R}>\mathrm{AI}=\mathrm{L}>\mathrm{CM}$ \\
\hline
\end{tabular}

Means \pm SD of the responses of the population of neurons recorded in each cortical area. Statistics: For min and peak lat, peak rate, FR index, and Int Index, the test was on multiple Kolmogorov-Smirnov tests. $<, P<0.01 ;=, P>0.01$. For all others, Monte Carlo analysis was used (see METHODS). $<, P<0.05$; $=, P>0.05$. Peak Act, peak activity; Min Lat, minimum latency; Peak Lat, peak latency; FR Index, firing rate index; Int Index, intensity index; BW10dB, bandwidth measured in octaves $10 \mathrm{~dB}$ above threshold; BW40dB, bandwidth $40 \mathrm{~dB}$ above threshold; Q10dB, Q measured $10 \mathrm{~dB}$ above threshold; Q40dB, Q measured $40 \mathrm{~dB}$ above threshold.

neurons had the shortest peak latencies and $\mathrm{R}$ neurons had the longest peak latencies.

\section{Intensity tuning}

Visual inspection of the firing rate as a function of stimulus intensity indicated that neurons could fall into three basic classes, similar to that described previously (Pfingst and

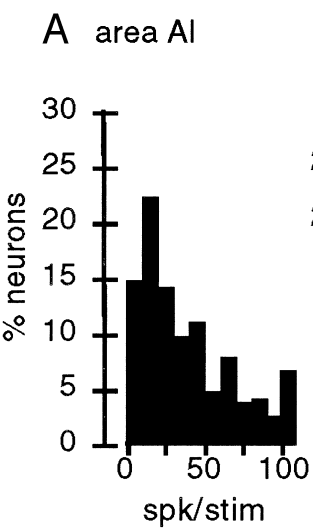

\section{$\mathrm{B}$ area $\mathrm{CM}$}
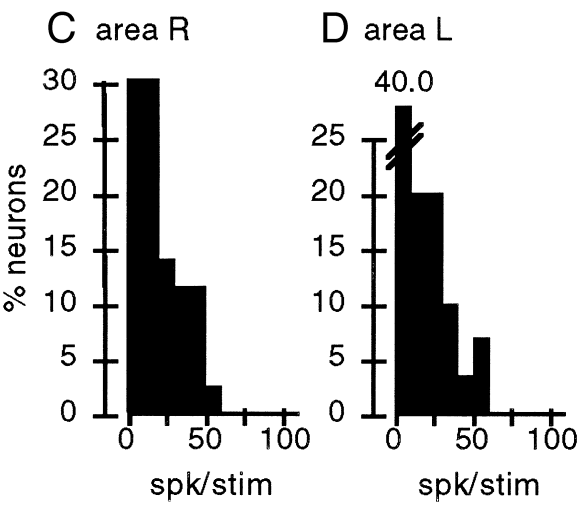

FIG. 9. Neuronal peak activity. A: frequency distribution of the peak activity for all AI neurons. Peak activity was taken as the stimulus that gave the greatest average response at the 5 frequencies tested closest to the $\mathrm{CF}$. $B$ : distribution of peak activity of $\mathrm{R}$ neurons. $C$ : $\mathrm{CM}$ neurons. $D$ : $\mathrm{L}$ neurons. Populations of AI and CM neurons had the greatest peak activity, and R neurons had the lowest peak activity
O'Connor 1981). Neurons could have firing rates that increased with increasing intensity (monotonic), other neurons showed increased firing rates with increased intensity up to an intermediate level and then a constant firing rate with increased intensity, and finally some neurons showed a peak in the firing rate at a particular stimulus intensity with decreased firing rates at higher and lower intensities (nonmonotonic). These rate/ level functions were quantified by defining the firing rate index by dividing the activity measured for the stimulus at the highest intensity tested by the highest level of activity elicited at any intensity (see METHODS). This index is 1.0 for neurons with monotonic rate/level functions and $<1.0$ for neurons with nonmonotonic rate/level functions. There was no significant linear or second-order correlation between the firing rate index and $\mathrm{CF}$ of $\mathrm{AI}$ neurons $(P>0.05)$. The distribution of the firing rate index across neurons in the different cortical areas is shown in Fig. 11A. Neurons in $\mathrm{R}$ had the lowest firing rate index, indicating that they had the most nonmonotonic rate/ level functions compared with the neurons in the other three areas $(P<0.01)$. Neurons in AI, L, and CM did not have firing rate indexes that were significantly different from each other $(P<0.01)$.

The firing rate index does not give an indication of the dynamic range over which the neuron is sensitive to changes in intensity. To address this point, the intensity index was calculated as the intensity (in $\mathrm{dB}$ SPL) that elicited the greatest response divided by the highest intensity tested. This index varies from 1.0 for neurons with monotonic rate/level functions to values of less than one depending on the intensity that produced the greatest response. The intensity index was lowest for $\mathrm{R}$ neurons compared with those in the other three cortical fields (Fig. 11B). The population of L neurons had significantly greater intensity indexes than those measured in the other three fields $(P<0.01)$, but there was no difference between neurons in $\mathrm{AI}$ and $\mathrm{CM}(P>0.05)$.

The stimulus intensity that elicited the greatest response was defined as the best intensity (Fig. 11C). Neurons in $\mathrm{R}$ had the lowest best intensities, on average $\sim 50 \mathrm{~dB}$ SPL, whereas AI and CM neurons had a average best intensities of $\sim 60 \mathrm{~dB}$ SPL. Neurons in the lateral region had the highest best intensities, at $\sim 65 \mathrm{~dB}$ SPL.

In summary, the analysis of intensity tuning indicated that 
A Al Neurons
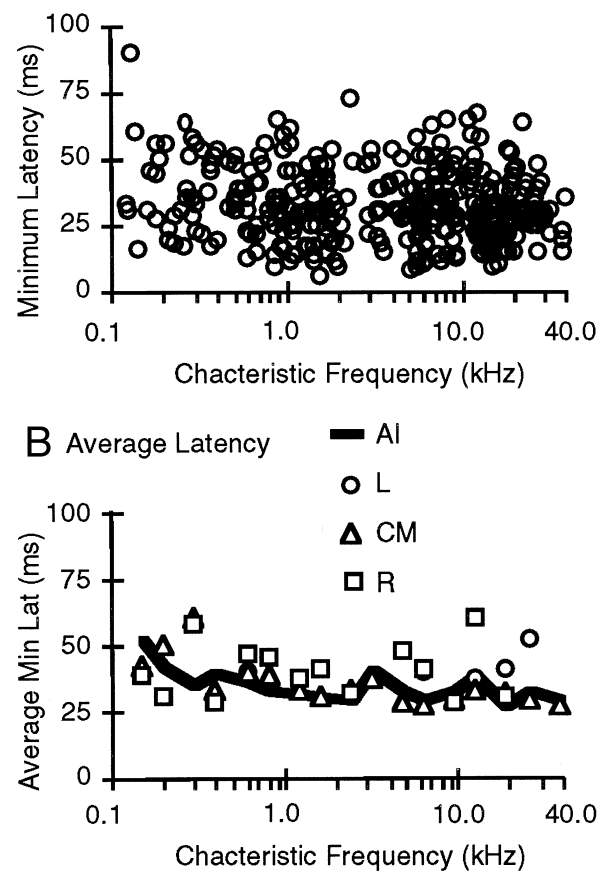

C Shortest Latency

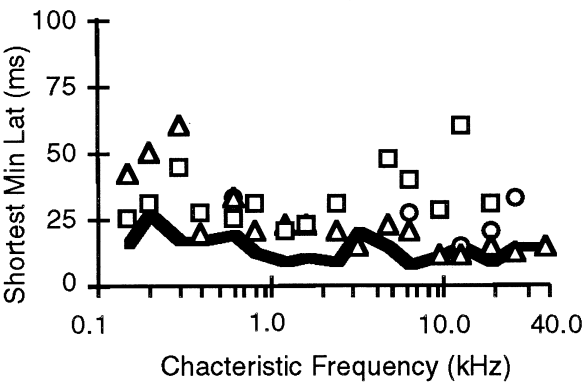

FIG. 10. Minimum latencies measured across neurons. A: minimum latencies measured for all AI neurons as a function of CF. Conventions as in Fig. $8 A$. $B$ : averaged minimum latencies for neurons in each of the 4 cortical areas tested. Neurons in the rostral field $(\square)$ consistently had longer minimum latencies that in the other cortical areas. Conventions as in Fig. 8B. C: shortest minimum latencies for neurons in each of the 4 cortical areas. Neuron with the shortest latency for each one-half octave band of CF is plotted. AI neurons consistently had the shortest latencies. Conventions as in Fig. $8 B$.

area $\mathrm{R}$ neurons had the most nonmonotonic rate/level functions and area $\mathrm{L}$ neurons had the most monotonic rate/level functions. These differences resulted in the lowest best intensities for neurons in $\mathrm{R}$ and the highest best intensities for neurons in $\mathrm{L}$. There was no difference in the best intensities or rate/level functions between neurons in AI and CM.

\section{Frequency tuning}

Two different measures were used to define the frequency tuning bandwidth at 10 and $40 \mathrm{~dB}$ above threshold. Each of the two measures of frequency tuning at both 10 and $40 \mathrm{~dB}$ above threshold showed a second-order correlation with CF $(P<$ 0.05). The first measure used the difference between the base 2 logarithm of the high- and low-frequency edges of the FRA relative to the characteristic frequency. The measure was arbitrarily assigned a value of 0.015 for neurons in which only one frequency elicited a driven response at 10 and/or $40 \mathrm{~dB}$ above threshold as the upper and lower edges could not be defined. Values of 1.0 indicate a bandwidth of one octave. The bandwidth measured at $10 \mathrm{~dB}$ above threshold (BW10) is shown for all AI neurons in Fig. 12A. Neurons with a CF near $10 \mathrm{kHz}$ had the narrowest bandwidths with wider bandwidths observed for neurons with higher and lower CFs. Neurons in CM consistently had the widest bandwidths and neurons in $\mathrm{R}$ had the narrowest bandwidths at $10 \mathrm{~dB}$ above threshold (Fig. 12B) compared with neurons in $\mathrm{AI}$ and $\mathrm{L}$.

A similar result was noted for frequency bandwidths measured $40 \mathrm{~dB}$ above threshold (Fig. 12, $C$ and $D$ ). Neurons in $\mathrm{CM}$ had the widest bandwidths, and neurons in $\mathrm{R}$ had the narrowest bandwidths. Neurons in the lateral field also generally had wider bandwidths than those found in AI, but this difference did not reach statistical significance. This is likely due to the nature of the FRAs of neurons in the lateral field, as there were commonly several frequencies with low-intensity responses, and only the lowest intensity response was considered in the preceding analysis (see Fig. 3, $A$ and $G$ ).

To compare these neuronal responses with those measured in other species, we also calculated the $Q$ value at 10 and $40 \mathrm{~dB}$ above threshold (e.g., Recanzone et al. 1999). This measure was taken as the CF divided by the bandwidth in kilohertz with larger numbers indicating more narrowly tuned neurons. For this analysis, neurons that had $Q$ values measured $>1,000$ (for

\section{A Firing Rate Index}

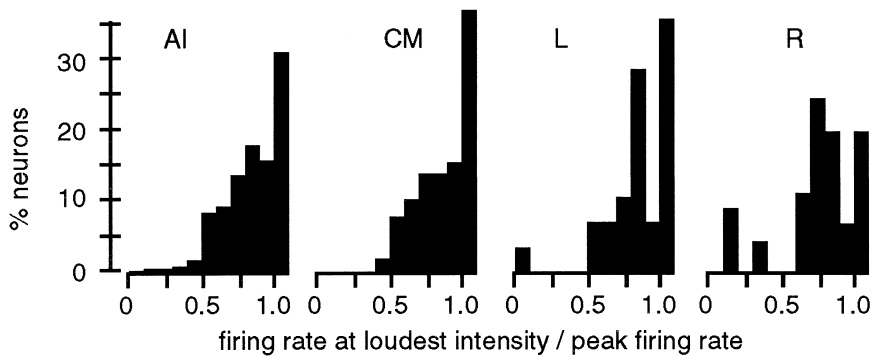

B Intensity index

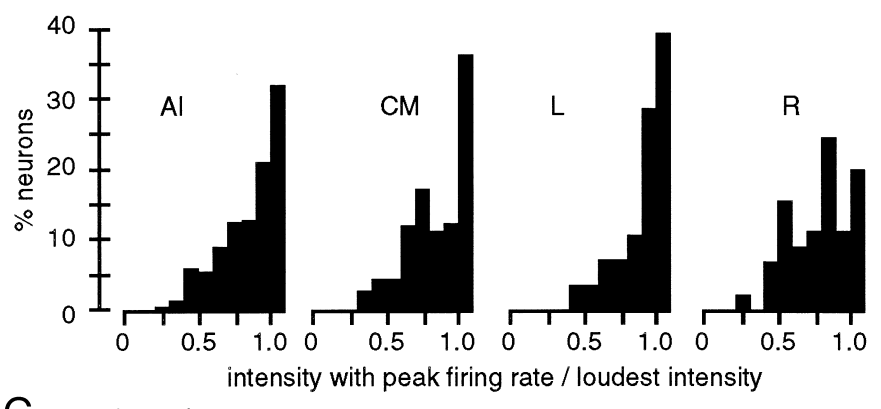

C Best Intensity

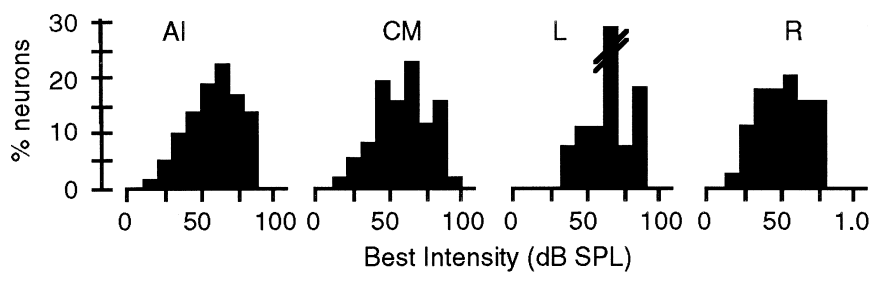

FIG. 11. Distributions of intensity tuning measures. $A$ : frequency distributions of the firing rate index for neurons in each of the 4 cortical areas. $B$ : frequency distributions of the intensity index and best intensity for neurons $(C)$ in each of the 4 cortical areas. 
A Single Neurons - BW $10 \mathrm{~dB}$

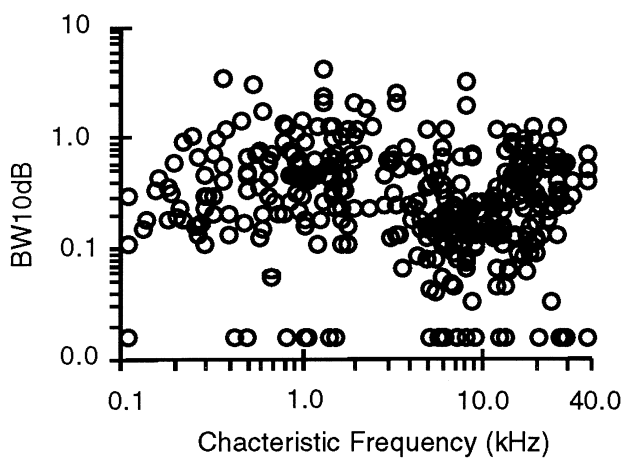

E Single Neurons - Q 10dB

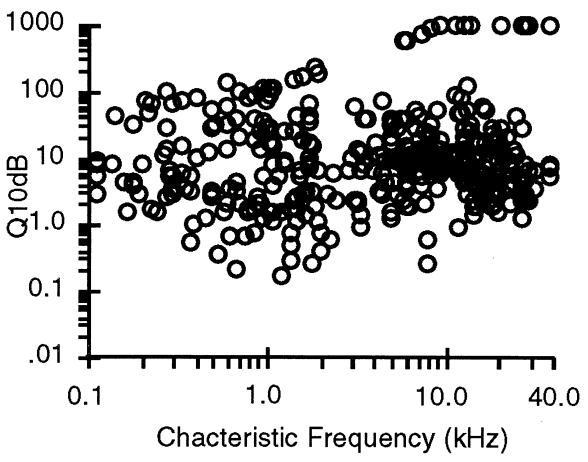

$F$ Q 10dB

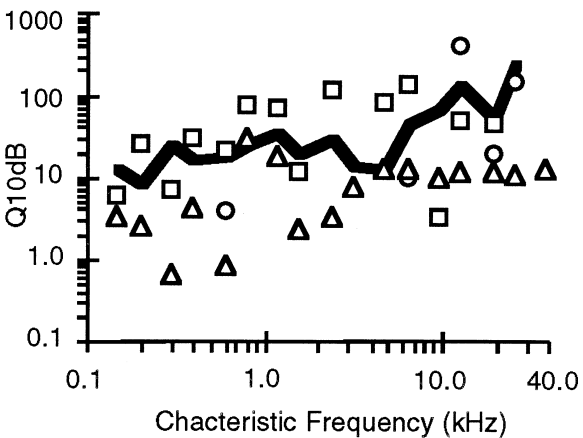

G Single Neurons - Q 40dB

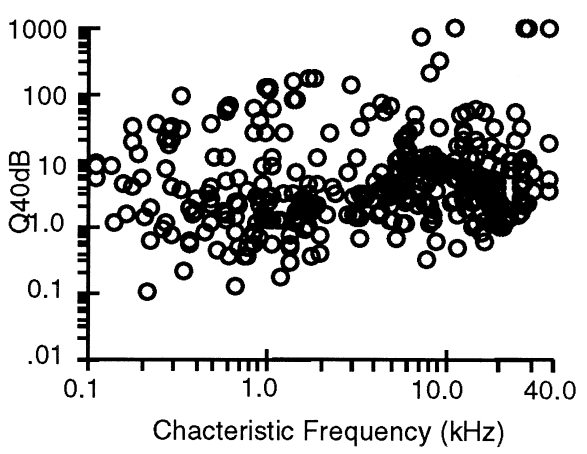

$H$ Q $40 \mathrm{~dB}$

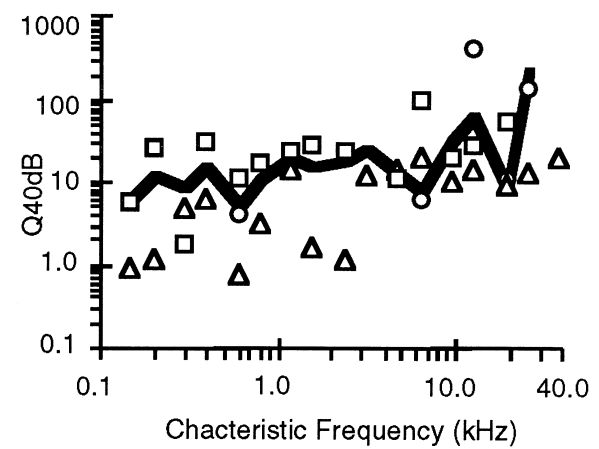

Chacteristic Frequency $(\mathrm{kHz})$
FIG. 12. Distributions of frequency bandwidth parameters. A: bandwidth measured 10 $\mathrm{dB}$ above threshold for all AI neurons. Neurons in which only the $\mathrm{CF}$ frequency gave a response were assigned a value of 0.015 . Conventions as in Fig. 8A. B: mean bandwidth measured $10 \mathrm{~dB}$ above threshold as a function of $\mathrm{CF}$ for neurons in all 4 cortical areas. Neurons in $\mathrm{CM}(\triangle)$ consistently had the highest bandwidths, whereas neurons in $\mathrm{R}(\square)$ consistently had the lowest bandwidths. Conventions as in Fig. $8 B$. $C$ : bandwidth measured $40 \mathrm{~dB}$ above threshold for all AI neurons. $D$ : mean bandwidth measured $40 \mathrm{~dB}$ above threshold as a function of $\mathrm{CF}$ for all neurons in all 4 cortical areas. $E$ : Q10 $\mathrm{dB}$ above threshold for all AI neurons. Neurons in which the Q10 measure exceeded 1,000 were assigned a value of $1,000 . F$ : mean Q $10 \mathrm{~dB}$ above threshold as a function of characteristic frequency for neurons in all 4 cortical areas. $G$ : Q40 dB above threshold for all AI neurons. $H$ : mean Q40 dB above threshold as a function of $\mathrm{CF}$ for all neurons in all 4 cortical areas. Neurons in CM $(\triangle)$ consistently had the lowest Q values (broadest tuning), whereas neurons in $R$ ( $\square$ ) consistently had the highest Q values (narrowest tuning).
BW $40 \mathrm{~dB}$

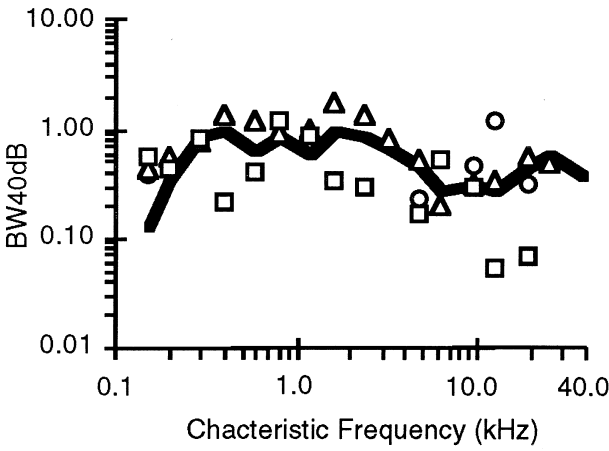

example neurons with a driven response only at the $\mathrm{CF}$ frequency at that intensity) were assigned arbitrarily a value of 1,000. Results of this analysis for intensities $10 \mathrm{~dB}$ above threshold are shown in Fig. 12, $E$ and $F$, and results for intensities $40 \mathrm{~dB}$ above threshold are shown in Fig. 12, $G$ and
$H$. The $Q$ values were lowest in CM (indicating more broadly tuned neurons) and greatest in $\mathrm{R}$ (indicating more narrowly tuned neurons) compared with neurons in AI (all $P$ values $<0.05)$. Neurons in the lateral field were not significantly different from those in AI. 
A Latency

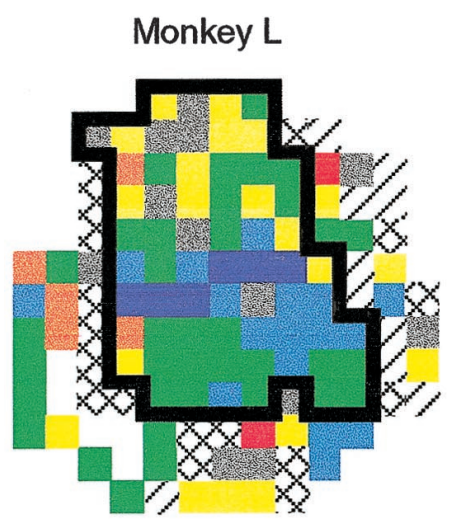

C Peak Activity

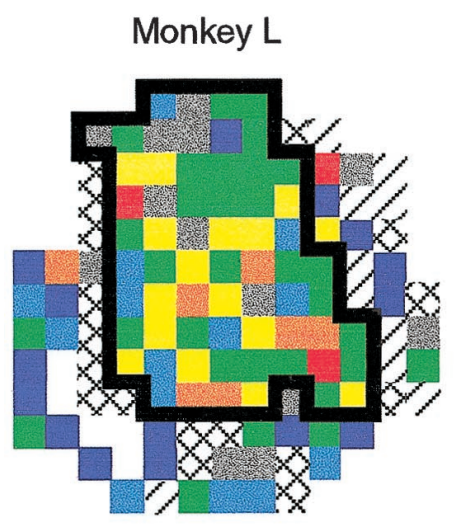

E Q40dB

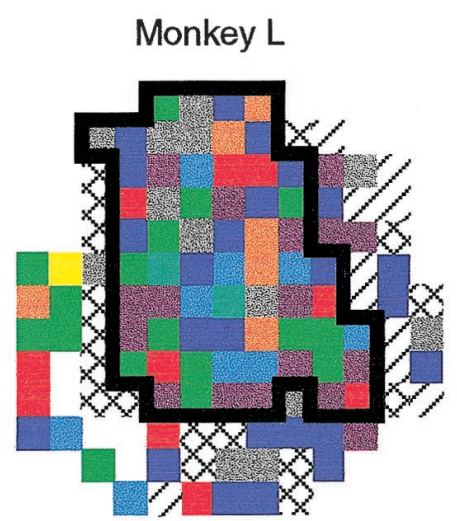

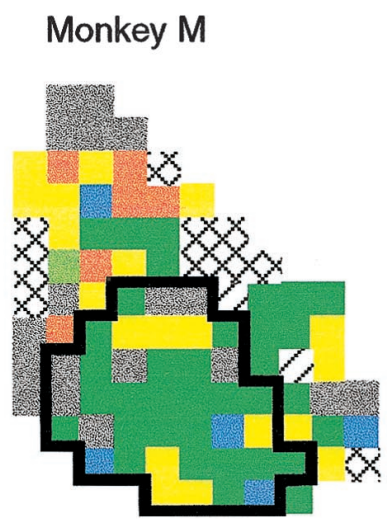

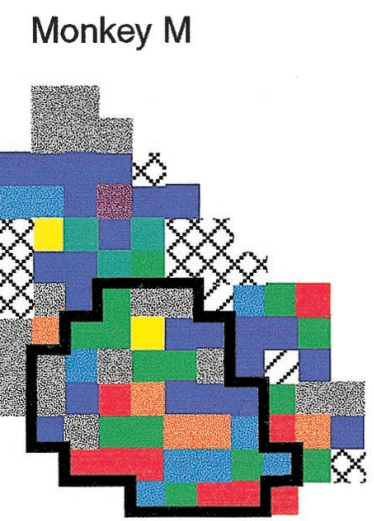

B Threshold

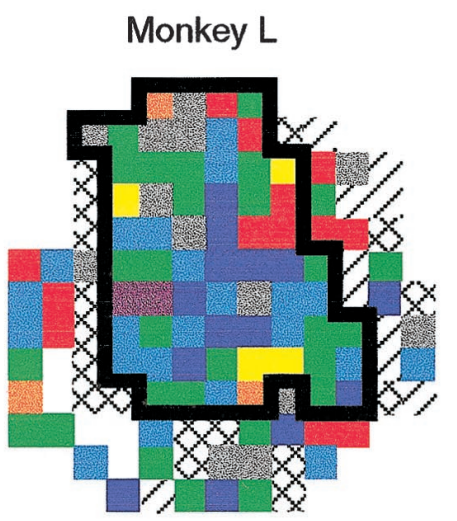

Monkey M

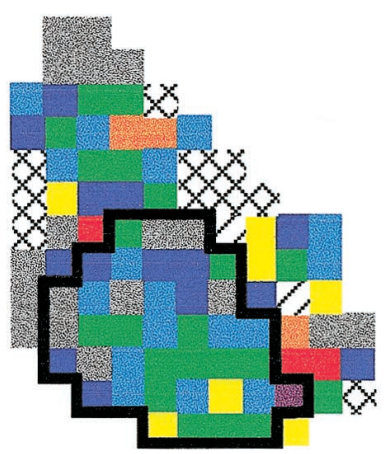

D Firing Rate Index

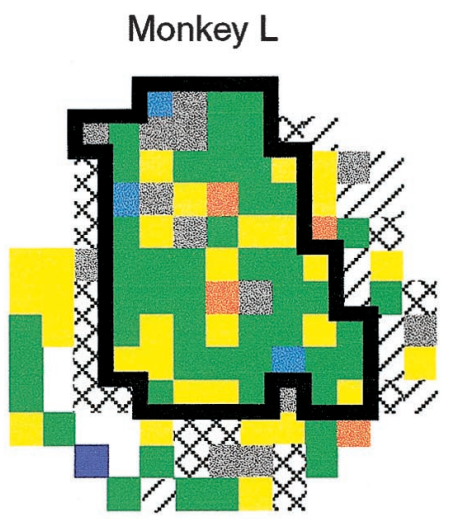

Monkey M

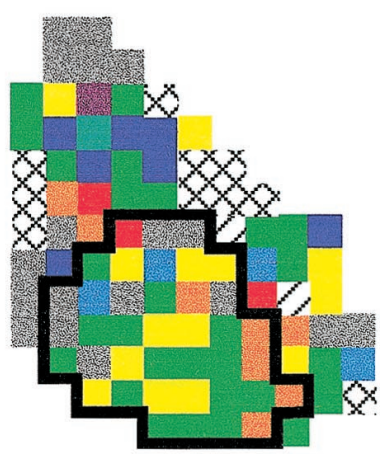

FIG. 13. Distribution across the spatial extent of auditory cortex for 5 different response parameters in both monkeys. Each plot shows the difference between the mean value of all single neurons measured at each location from the mean value measured across AI neurons sharing the same $\mathrm{CF}$ for each parameter illustrated. Percent differences are coded by color (see legend). Gray squares correspond to locations where the response parameter was not quantified. A: minimum latency measured in monkey L (left) and monkey $M$ (right). B: threshold. C: peak response. D: firing rate index. E: Q40.

In summary, the frequency bandwidth measures at both 10 and $40 \mathrm{~dB}$ above threshold indicated that the most broadly tuned neurons were found in $\mathrm{CM}$ and the most narrowly tuned neurons were found in R. Although neurons in the lateral field generally had more broadly tuned responses, they were not statistically significantly different from those found in AI.

\section{Spatial distribution of response parameters}

Previous studies have shown that different response properties are distributed systematically and nonrandomly across AI in the anesthetized owl monkey (Recanzone et al. 1999) and cat (e.g., see Schreiner et al. 1998). To determine if this is also the case in the awake macaque monkey, we investigated the 
A Al neurons
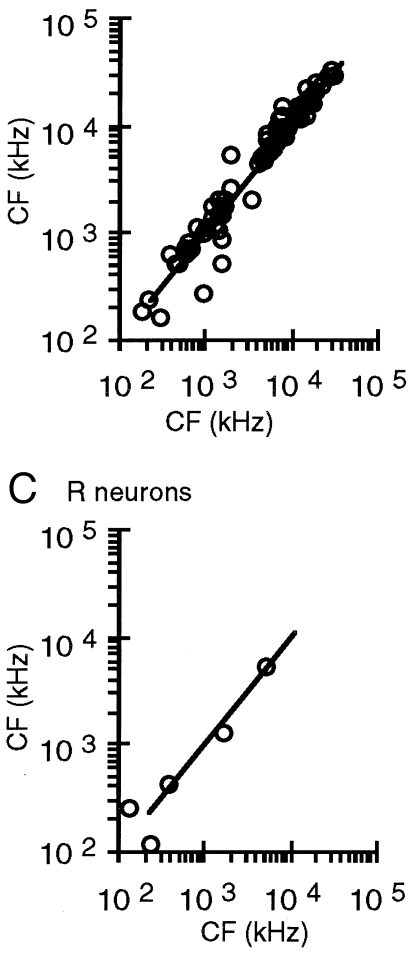

$B$ L neurons

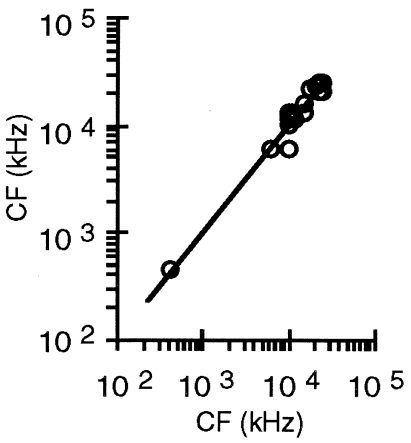

D CM neurons

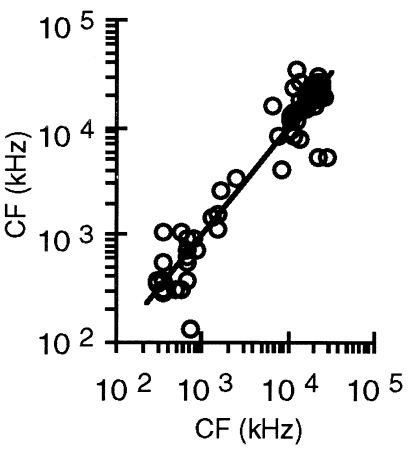

FIG. 14. Variability of CF between neurons recorded simultaneously from the same electrode. Each plot shows the CF measured between 1 ( $x$ axis) and a 2 nd ( $y$ axis) neuron. $A$ : comparisons of AI neurons $(n=101) . B$ : L neurons $(n=19)$. $C$ : R neurons $(n=5) . D$ : CM neurons $(n=68)$.

spatial distribution of all of the 11 response parameters shown in Table 2. To directly compare the spatial distributions between monkeys and response parameters, all values were normalized to the mean responses measured in the AI neurons, taking into account the $\mathrm{CF}$ of the neurons for the latency, threshold, and frequency tuning measures. These values then were plotted as a percentage of this mean, with five representative examples shown in Fig. 13.

Neurons within AI showed little systematic organization and considerable variability between monkeys for all of the response parameters measured. In each of the illustrated cases, as well as for the other response parameters not shown, there was no systematic organization that was consistent between monkeys. For example, the neurons with the lowest thresholds in monkey $L$ were near the center of the medial-lateral extent of AI, but this did not hold true for monkey $M$ (Fig. 13B). Figure $13 E$ shows the results from the analysis of Q40, where a systematic organization was observed across owl monkeys (Recanzone et al. 1999) and cats (e.g., Schreiner and Sutter 1992) yet was not apparent in these two behaving monkeys.

One possible source of the lack of any obvious internal topography could be that the responses at each recording location were based on the average of the single neuron responses. The preceding analysis showed considerable variability across neurons with similar CFs for all parameters studied, and presumably this included neurons at the same or very nearby locations (see also Table 2). The degree of variability between adjacent neurons was analyzed for each response parameter by regression analysis between two different neurons recorded at the same time through the same electrode.
This analysis is shown for CF in Fig. 14. Although some differences occasionally were noted, for neurons in all cortical areas there was a strong correlation of the CF between the two neurons. The correlation coefficients are shown in Fig. 15 for nine of the response parameters tested. For neurons recorded in AI, there was a significant correlation $(P<0.05)$ for all response properties. However, for all parameters other than $\mathrm{CF}$, the regression coefficients were $<0.6$, indicating that there was considerable variability in the response between adjacent neurons.

The correlation coefficients for $\mathrm{CF}$ were the greatest for all response parameters measured across cortical areas, indicating the least amount of variability for this parameter. Neurons in L had high correlation coefficients for the intensity tuning pa-
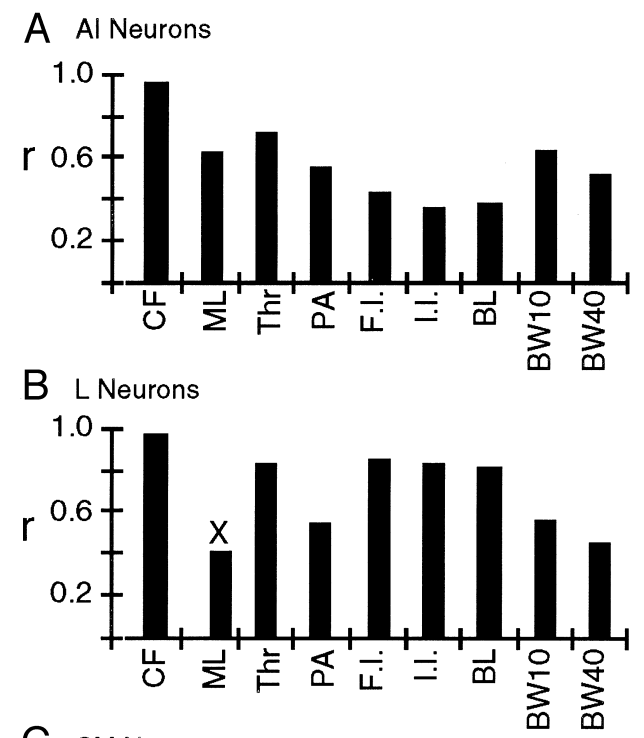

C CM Neurons

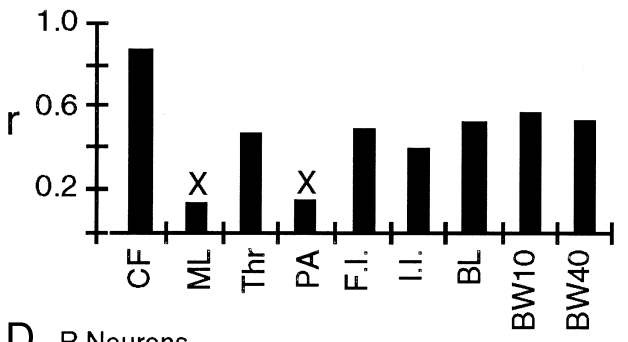

D R Neurons

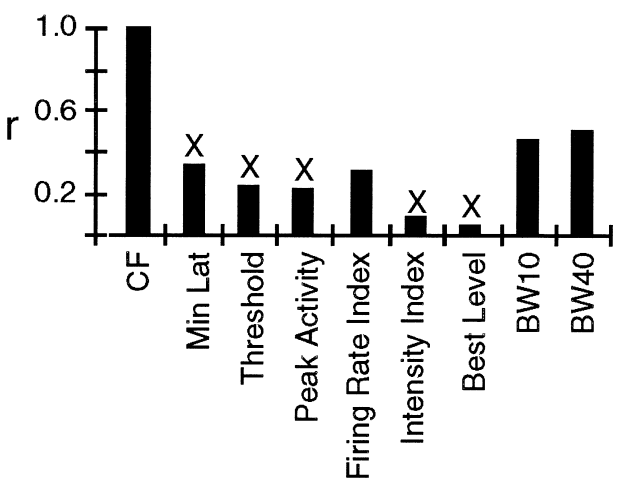

FIG. 15. Correlation coefficients across response parameters. Each plot shows the correlation coefficients for each of 9 response parameters for the neurons shown in Fig. 14. $\times$, correlations that were not statistically significant $(P<0.05)$. $A$ : AI neurons. $B$ : $\mathrm{L}$ neurons. $C$ : $\mathrm{CM}$ neurons. $D$ : $\mathrm{R}$ neurons. 
TABLE 3. Early versus late response properties

\begin{tabular}{lrccccc}
\hline \hline Parameter & $0-100 \mathrm{~ms}$ & $25-65 \mathrm{~ms}$ & $65-100 \mathrm{~ms}$ & $t$-test & $r$ & Slope \\
\hline CF & $138-27,000$ & $185-23,000$ & $150-25,000$ & $>0.05$ & 0.982 & 0.989 \\
Threshold & $22 \pm 13.5$ & $35.1 \pm 14.3$ & $40.8 \pm 24.1$ & $*$ & 0.710 & 1.192 \\
Peak Act. & $16.54 \pm 7.81$ & $16.29 \pm 8.11$ & $10.03 \pm 2.22$ & $* *$ & 0.787 & 0.216 \\
F.R. Index & $0.77 \pm 0.25$ & $0.973 \pm 0.81$ & $0.839 \pm 0.25$ & $* *$ & 0.147 & 0.472 \\
Int. Index & $0.98 \pm 0.11$ & $0.944 \pm 0.118$ & $0.868 \pm 0.186$ & $*$ & 0.229 & 0.359 \\
Best Intens. & $53.7 \pm 16.4$ & $84.9 \pm 10.6$ & $78.2 \pm 16.8$ & $*$ & 0.229 & 0.359 \\
BW10dB & $0.66 \pm 0.44$ & $1.01 \pm 0.733$ & $0.924 \pm 0.626$ & $>0.05$ & 0.611 & 0.528 \\
BW40dB & $0.93 \pm 1.01$ & $2.38 \pm 1.52$ & $2.07 \pm 1.44$ & $>0.05$ & 0.814 & 0.749 \\
\hline
\end{tabular}

Means \pm SD of the 41 neurons tested using different response periods. Times are relative to the onset of the 50 -ms stimulus. Characteristic frequency (CF)

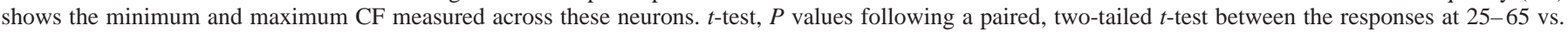

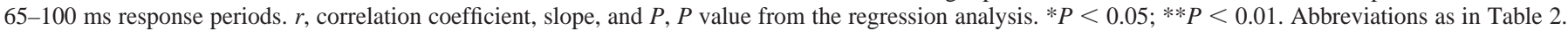

rameters, consistent with their monotonic rate/level functions, whereas neurons in CM had high correlation coefficients for the frequency tuning parameters, consistent with having the widest frequency tuning bandwidth for neurons across all cortical areas (see Table 2). For area $\mathrm{R}$ neurons, the sample size was too small to make any definitive conclusions $(n=5)$, but these data were included in Fig. 15 for comparison. These results indicate that within a given cortical area, the response parameter that was the most different compared with the other cortical areas (i.e., frequency bandwidth in CM or intensity tuning in L) showed the least amount of variability compared with other response parameters within that cortical area.

\section{Temporal response characteristics}

A final consideration is the potential differences in the responses between different time periods relative to the onset of the stimulus. Some auditory cortical neurons in the awake monkey have clear offset responses in addition to, or exclusive of, onset responses (e.g., Pfingst and O'Connor 1981). The response properties of the neurons during the poststimulus period was directly related to the response during the stimulus period by comparing the responses during 25-65 ms from stimulus onset ("early") to 65-100 ms ("late"). These periods were chosen as none of these neurons had minimum latencies to the onset of the stimulus $<15 \mathrm{~ms}$. The majority of the neurons with responses to stimulus frequencies near CF during the late period had either no driven responses or low and highly variable firing rates, which made it impossible to effectively define the response parameters using the previous criteria. There were 40 AI neurons and $1 \mathrm{CM}$ neuron that had clearly defined frequency and intensity response areas based on the activity during the late period similar to those shown in Figs. $3-7$, and these neurons were further analyzed with respect to eight different response parameters (Table 3). There was no significant difference for $\mathrm{CF}, \mathrm{BW} 10$, or BW40 (all $P$ values $>0.05$; paired $t$-test), but there was a difference for the activity level parameters of threshold and peak activity, as well as the intensity tuning parameters of firing rate index, intensity index, and best intensity (all $P$ values $<0.05$; see Table 3 ). Regression analysis between the responses during these two stimulus periods showed that the threshold and peak activity was consistently different between the two response periods, whereas for the intensity tuning parameters, there was no significant correlation between the two response periods (Table 3 ).

The poststimulus responses of these neurons also were compared with the entire population of neurons using the $0-100 \mathrm{~ms}$ response period as described in the preceding text. Comparison between the mean responses across parameters between 0 and $100 \mathrm{~ms}$ and the shorter intervals showed clear differences (compare Tables 2 and 3). Neurons with poststimulus responses had lower thresholds, more monotonic rate/level functions, and wider frequency bandwidths compared with the overall population of AI neurons studied (all $P$ values $<0.05$ ). However, the main point of this analysis was to determine if the poststimulus responses would significantly alter the distributions of the response properties described in the preceding text. The replacement of the previous analysis with the poststimulus responses of these relatively few neurons resulted in no significant differences in the statistical analyses presented in the preceding text.

\section{I S C U S S I O N}

We report the first comprehensive survey of response properties to tonal stimuli recorded from single auditory cortical neurons in the behaving macaque monkey. The topographic representation of $\mathrm{CF}$ indicated that neurons were sampled from AI, R, CM, and the field lateral to AI which we have defined as L. Quantification of these response properties showed that neurons in $\mathrm{R}$ had the lowest activity levels, longest latencies, the most nonmonotonic rate/level functions, and the sharpest frequency tuning across neurons in the four cortical fields. Neurons in CM had the broadest frequency tuning and highest thresholds, and neurons in $\mathrm{L}$ showed the most monotonic rate/level functions. Interestingly, neurons in AI were intermediate in all response parameters tested, except that neurons with the shortest latencies were consistently found in AI compared with neurons in the other three cortical areas. We found little apparent systematic organization within a cortical field of any response parameter except for $\mathrm{CF}$. There was considerable variability in the responses between neurons recorded simultaneously from the same electrode. This variability was smallest for $\mathrm{CF}$ across all four cortical areas, and smallest for AI neurons compared with neurons in the other three cortical areas.

\section{Organization of auditory cortex}

Previous studies in the anesthetized macaque monkey have indicated that the primary auditory cortex is surrounded by at least three or more surrounding cortical fields. AI is characterized by short-latency neuronal responses to tonal stimuli with narrow frequency tuning and is organized with a CF gradient 
from high frequency to low frequency in the caudal to rostral direction (Kosaki et al. 1997; Merzenich and Brugge 1973; Morel et al. 1993; Rauschecker et al. 1997). Anatomically this region corresponds to koniocortex, is stained densely with cytochrome oxidase histochemistry and for parvalbumin immunoreactivity (Jones et al. 1995; Merzenich and Brugge 1973; Morel et al. 1993), and receives the majority of its thalamic input from the ventral division of the medial geniculate nucleus (Hashikawa et al. 1995; Molinari et al. 1995; Morel et al. 1993; Rauschecker et al. 1997).

We measured neuronal CFs between $\sim 100 \mathrm{~Hz}$ to almost 40 $\mathrm{kHz}$, a slightly broader range than described in anesthetized monkeys (Kosaki et al. 1997; Rauschecker et al. 1997). Quantitative measures of the frequency tuning were similar to the Q10 measure in the anesthetized monkey (Kosaki et al. 1997), and the latencies were similar, although in some cases shorter to those reported previously in the awake monkey (Pfingst and O'Connor 1981). Thresholds measured in these neurons were consistent with a smaller sample of neurons reported by Pfingst et al. (1977) and with the behaviorally measured audiograms from macaques (Owren et al. 1988; Stebbins 1966). We also observed a range of intensity tuning functions, similar to but not as sharply intensity tuned as those described previously (Pfingst and O'Connor 1981).

Rostrally, a second field shows a reversal of the CF gradient and has been termed RL (Merzenich and Brugge 1973) or R (Morel et al. 1993). This region is nearly indistinguishable from AI based on cytoarchitecture and immunoreactivity but shows a different pattern of cytochrome oxidase staining (Jones et al. 1995; Merzenich and Brugge 1973; Morel et al. 1993). We recorded neurons rostral to AI that were similar with respect to the CF progression and frequency tuning compared with previous reports in both the anesthetized (Kosaki et al. 1997; Merzenich and Brugge 1973; Morel et al. 1993; Rauschecker et al. 1997) and awake macaques (Pfingst and O'Connor 1981). The latencies were significantly longer than those in AI, which was suggested previously based on a much smaller number of neurons (Pfingst and O'Connor 1981). A novel finding from this study was the greater percentage of intensity tuned neurons compared with AI, which represents a clear physiological difference between these two areas. A second difference we noted was in the peak activity level to these tonal stimuli, with neurons in $\mathrm{R}$ showing lower activity levels to these tone stimuli compared with neurons in the other cortical fields. This result indicates that neurons in $\mathrm{R}$ would likely respond with greater activity if a different stimulus had been used (see following text).

Caudal and medial to AI is CM, which has been described as nontonotopic and can be distinguished from AI and R by cytoarchitecture, immunoreactivity, and connections with the medial geniculate nucleus (Kosaki et al. 1997; Merzenich and Brugge 1973; Morel et al. 1993; Rauschecker et al. 1997). Some investigators have suggested that this field is either relatively small or nonexistent along the medial region of AI (e.g., Jones et al. 1995), whereas others have noted this field located medial to AI (e.g., Morel et al. 1993). In both monkeys of the present study, we recorded neurons medial to AI that were physiologically distinct from $\mathrm{AI}$ and thus classified as area CM. The responses of neurons recorded in CM were distinguished by their significantly broader frequency tuning compared with neurons in the other cortical fields, consistent with previous qualitative observations in the anesthetized monkey (e.g., Merzenich and Brugge 1973; Morel et al. 1993; Rauschecker et al. 1997). We also found that these neurons had the highest thresholds compared with neurons in the other cortical fields but were similar to neurons in AI with respect to the peak activity level and the intensity tuning functions. This is consistent with recent studies on the dependence of $\mathrm{CM}$ on AI neurons for responding to tonal stimuli (Rauschecker et al. 1997) and further indicates that CM neurons require inputs from multiple AI neurons to respond to tonal stimuli.

Finally, neurons lateral to AI (L) have been described to have broad tuning and a distinct anatomic organization, including projections from the thalamus (Kosaki et al. 1997; Merzenich and Brugge 1973; Morel et al. 1993; Pfingst and O'Connor 1981; Rauschecker et al. 1997). Neurons in the lateral field show systematic reversals in the $\mathrm{CF}$ gradient (Rauschecker et al. 1995), suggesting at least three distinct functional subdivisions termed AL, ML, and CL (Rauschecker 1998). The neurons we recorded were lateral to AI and likely correspond to the medial lateral field (ML), although there was not a clear tonotopic organization. Neurons in this area had similar thresholds and frequency tuning to neurons in AI but could be distinguished by the higher proportion of neurons with monotonic rate/level functions.

\section{Technical considerations}

We have relied on the $\mathrm{CF}$ and frequency tuning parameters to categorize neurons at each recording location into the different cortical fields in these monkeys. Anatomic verification is not yet possible as both of these monkeys are currently participating in experiments; however, our definitions of these cortical areas are consistent with previous studies in anesthetized and awake animals, and the MRI images taken from monkey $L$ are consistent with the gross morphology of the primary and surrounding auditory cortical fields (e.g., Jones et al. 1995; Merzenich and Brugge 1973). In a few cases, it is possible that the classification of neurons at the border regions may be in error, for example between $\mathrm{CM}$ and $\mathrm{L}$ in monkey $L$ or between $\mathrm{AI}$ and $\mathrm{R}$ in monkey $M$, but this limited number of neurons would likely not affect the main results or our interpretations, as evidenced by the fact that restricting the analysis, at least with respect to the population of $\mathrm{R}$ neurons, to neurons that did not form part of the AI border showed the equivalent results.

One concern is that our samples of neurons in $\mathrm{R}$ and $\mathrm{L}$ were relatively small and were taken from a single monkey. We found no statistically significant differences in any response property between the two monkeys for neurons located in either AI or CM, and therefore we believe that our observations reflect real differences in the response properties of neurons in $\mathrm{R}$ and $\mathrm{L}$ compared with the other cortical fields. However, the smaller sample size could have been more sensitive to the variability we observed in most response parameters between neurons within a given cortical field, causing a bias in the results. Nonetheless the findings of this study did stand up to statistical tests, and we believe indicate real functional differences between neurons in these different cortical areas.

Part of the differences in response properties between cortical areas may be due to the use of brief tonal stimuli, which are likely ineffective in driving neurons in the nonprimary cortical areas (e.g., see Rauschecker et al. 1995, 1997). For 
example, the latency of the response is generally shortest for stimuli that elicit the best response (see Pfingst and O'Connor 1981), and if we had chosen a more effective stimulus in driving the nonprimary cortical neurons, the latency and thresholds may have been more similar to those found in AI. The definition of exactly what stimulus best drives these neurons remains to be determined.

\section{Internal organization of AI}

A difference between the results of this study and findings from both the anesthetized cat and owl monkey is the lack of any apparent systematic representation of the different response properties. Studies in the cat have revealed a systematic organization within AI for the frequency tuning bandwidth (Schreiner and Mendelson 1990; Schreiner and Sutter 1992), intensity tuning (Heil et al. 1994; Schreiner et al. 1992; Sutter and Schreiner 1995), response latency (Mendelson et al. 1997) responses to frequency-modulated sweeps (Mendelson et al. 1993) and human speech segments (Schreiner 1998). A recent study in the anesthetized owl monkey similarly showed nonrandom distributions within AI of Q10, Q40, best intensity, latency, and threshold (Recanzone et al. 1999). It was suggested that this internal topography may be common across species and represent a basic organizational feature of AI. The results of the current study indicate that, at least in the awake macaque monkey, such a basic organizational feature is not apparent.

There are several reasons why the techniques used in the present report could fail to reveal the existence of a systematic organization similar to that seen in the anesthetized preparation. The first is in the difference between using multiple-unit recording techniques and averaging the responses of single neurons at each cortical location (i.e., see Sutter and Schreiner 1995). For example, in a multiple-unit recording the minimum latency would reflect the neuron with the shortest latency in the cluster compared with the average latency for all neurons recorded at that location. Similar differences would be noted for most of the response parameters we tested. In addition, previous studies in anesthetized animals recorded from neurons located primarily in the middle cortical layers, whereas our sample likely included neurons from other cortical layers as well. Finally, anesthetics may reduce the variability of the responses and therefore increase the ability to observe a systematic organization. Clear differences have been observed in the responses of auditory cortical neurons among attending, unattending, and anesthetized states in the monkey, but the degree of these attentional effects varied between neurons (e.g., Miller et al. 1972; Pfingst et al. 1977). These monkeys performed this relatively simple task with high efficiency, but the degree of motivation or attentional state could have fluctuated during the experiments. Whether these factors prevented us from observing topographic representations of different response characteristics across AI, or if no such topographic representation exists, will require future experiments directly addressing this issue.

\section{Functional role of auditory cortical areas}

It has been suggested that auditory information is processed by both serial and parallel pathways in auditory cortex (Kaas et al. 1999; Rauschecker 1998), similar to the ventral and dorsal processing streams described in the visual system (Ungerleider and Haxby 1998; Ungerleider and Mishkin 1982). Anatomic evidence supports the notion of multiple auditory processing pathways from the thalamus through the cortex, and the results of the present study lend further support for serial processing of auditory information. We found that AI neurons had intermediate values of all response parameters (except that neurons with the shortest latencies were in $\mathrm{AI}$ ) and the least variability between neighboring neurons for all response parameters. Neurons in other cortical areas that had the highest or lowest values of a response parameter, for example, frequency tuning bandwidth in CM or intensity tuning measures in L, showed the lowest amount of variability for that parameter. This is expected if neurons in the nonprimary areas are integrating information from a subset of AI neurons, thereby decreasing the variability in the responses for the parameter of interest and increasing the variability for other response parameters.

The similarities between $\mathrm{AI}$ and $\mathrm{R}$ using anatomic criteria and qualitative physiological observations prompted the question of the functional differences between neurons in these two regions. We have found that neurons in $\mathrm{R}$ have significantly longer latencies, have lower peak activity levels, and have sharper intensity tuning than neurons in AI. Given the intensity tuning of these neurons, it is likely that they are more strongly influenced by inhibitory interneurons and would be well suited to process stimuli with local variations in frequency and intensity, such as harmonic complexes.

Neurons in the lateral field were more broadly tuned than AI neurons and showed the highest proportion of intensity insensitive neurons. Previous studies have shown that these neurons respond better to more complex stimuli, such as band-passed noise and vocalizations (Rauschecker et al. 1995). Anatomically, these neurons project predominately to the more lateral fields, termed the para belt region, which in turn project to more temporal regions of cortex (Hackett et al. 1998a,b). This evidence has led to the suggestion that lateral field neurons form part of a ventral pathway that processes information regarding the identification of acoustic stimuli (Rauschecker 1998). Our results are consistent with this idea and showed that neurons in this area have the most monotonic rate/level functions, which would allow processing of such complex stimuli across a large range of stimulus intensities.

Finally, it has been suggested, primarily based on the broad frequency tuning and anatomic connections, that neurons in CM play a role in sound localization (Rauschecker 1998). The results of the present study have quantitatively shown that CM neurons have the broadest frequency tuning and highest thresholds but were similar to AI neurons in most other respects. This broad tuning could be useful in processing the spectral cues necessary to process sound location information, and indeed many of these neurons show spatial selectivity that is better correlated with sound localization performance compared with AI neurons (Recanzone et al. 2000).

Taken together, it seems reasonable that acoustic signal processing occurs in both a parallel and serial fashion throughout the auditory cortex. The results of this study indicate that these four cortical fields can be distinguished on physiological grounds and suggest that each of these cortical areas likely have very different functional roles. The exact nature of the function of these areas is currently poorly understood, but it 
appears clear that the use of more complex acoustic stimuli in the behaving monkey will provide valuable insights into the functional role of these different cortical areas within the auditory cortex.

The authors thank M. L. Sutter, T. M. Woods, and T. K. Su for helpful comments on earlier versions of this manuscript, M. N. Ortigas-Neefe and T. K. Su for participating in these experiments, and the California Regional Primate Research Center for expert veterinary care.

This work was funded by National Institute on Deafness and Other Communication Disorders Grant DC-02371, The Klingenstein Fund, and the Sloan Foundation (all to G. H. Recanzone).

Address for reprint requests: G. H. Recanzone, Center for Neuroscience, 1544 Newton Ct., Davis, CA 95616.

Received 19 November 1999; accepted in final form 7 January 2000.

\section{REFERENCES}

Ahissar, M., Ahissar, E., Bergman, H., and VaAdia, E. Encoding of soundsource location and movement: activity of single neurons and interactions between adjacent neurons in the monkey auditory cortex. J. Neurophysiol. 67: 203-215, 1992.

Benson, D. A. and Hienz, R. D. Single-unit activity in the auditory cortex of monkeys selectively attending left vs. right ear stimuli. Brain Res. 59: 307-20, 1978.

Benson, D. A., Hienz, R. D., And Goldstein, M. H., JR. Single-unit activity in the auditory cortex of monkeys actively localizing sound sources: spatial tuning and behavioral dependency. Brain Res. 219: 249-267, 1981.

Cipolloni, P. B. And Pandya, D. N. Golgi, histochemical, and immunocytochemical analyses of the neurons of auditory-related corticies of the rhesus monkey. Exp. Neurol. 114: 104-122, 1991.

Crist, C. F., Yamasaki, S.D.G., Komatsu, H., and Wurtz, R. H. A grid system and a microsyringe for single cell recording. J. Neurosci. Methods 26: $117-122,1988$

Felleman, D. J. AND VAn Essen, D. C. Distributed hierarchical processing in the primate cerebral cortex. Cereb. Cortex 1: 1-47, 1991.

Hackett, T. A, Stepniewska, I., AND KaAs, J. H. Subdivisions of auditory cortex and ipsilateral cortical connections of the parabelt auditory cortex in macaque monkeys. J. Comp. Neurol. 394: 475-495, 1998a.

Hackett, T. A., Stepniewska, I., And KaAs, J. H. Thalamocortical connections of the parabelt auditory cortex in macaque monkeys. J. Comp. Neural. 400: 271-286, 1998b.

Hashikawa, T., Molinari, M., Ransell, E., and Jones, E. G. Patchy and laminar terminations of medial geniculate axons in monkey auditory cortex. J. Comp. Neurol. 362: 195-208, 1995.

HeIl, P., Rajan, R., and Irvine, D. R. Topographic representation of tone intensity along the isofrequency axis of cat primary auditory cortex. Hear. Res. 76: 188-202, 1994.

Hubel, D. H., Henson, C. O., Rupert, A., and Galambos, R. "Attention" units in the auditory cortex. Science 129: 1279-1280, 1959.

Jones, E. G., Dell, M. E., Molinari, M., Rausell, E., and Hashikawa, T. Subdivisions of macaque monkey auditory cortex revealed by calciumbinding protein immunoreactivity. J. Comp. Neurol. 362: 153-170, 1995.

KaAs, J. H., Hackett, T. A., and Tramo, M. J. Auditory processing in the primate cerebral cortex. Curr. Opin. Neurobiol. 9: 164-170, 1999.

Kosaki, H., Hashikawa, T., He, J., and Jones, E. G. Tonotopic organization of auditory cortical fields delineated by parvalbumin immunoreactivity in macaque monkeys. J. Comp. Neurol. 386: 304-316, 1997.

Mendelson, J. R., Schreiner, C. E., AND Sutter, M. L. Functional topography of cat primary auditory cortex: response latencies. J. Comp. Physiol. A Sens. Neural. Behav. Physiol. 181: 615-633, 1997.

Mendelson, J. R., Schreiner, C. E., Sutter, M. L., and Grasse, K. L. Functional topography of cat primary auditory cortex: responses to frequency-modulated sweeps. Exp. Brain Res. 94: 65-87, 1993.

Merzenich, M. M. AND BRUGGe, J. F. Representation of the cochlear partition on the superior temporal plane of the Macaque monkey. Brain Res. 50: 275-296, 1973.

Miller, J. M. Dobie, R. A., Pfingst, B. E., And Hienz, R. D. Electrophysiological studies of the auditory cortex in the awake monkey. Am. J. Otolaryngol. 1: 119-30, 1980.
Miller, J. M., Sutton, D., Pfingst, B., Ryan, A., Beaton, R., And GoureVITCH, G. Single cell activity in the auditory cortex of Rhesus monkeys: behavioral dependency. Science 177: 449-451, 1972.

Molinari, M., Dell'Anna, M. E., Rausell, E, Leggio, M. G., Hashikawa, T., AND JONES, E. G. Auditory thalamocortical pathways defined in monkeys by calcium-binding protein immunoreactivity. J. Comp. Neurol. 362: 171194, 1995.

Morel, A., Garraghty, P. E., and KaAs, J. H. Tonotopic organization, architectonic fields, and connections of auditory cortex in macaque monkeys. J. Comp. Neurol. 335: 437-459, 1993.

Mosteller, F., Fienberg, S. E., AND Rourke, R.E.K. Beginning Statistics with Data Analysis Reading. Reading, MA: Addison-Wesley, p. 328-367.

Owren, M. J., Hopp, S. L., Sinnott, J. M., And Petersen, M. R. Absolute auditory thresholds in three Old World monkey species (Cercopithecus aethiops, C. neglectus, Macaca fuscata) and humans (Homo sapiens). J. Comp. Psychol. 102: 99-107, 1988.

Pandya, D. N. And Rosene, D. L. Laminar termination patterns of thalamic, callosal, and association afferents in the primary auditory area of the rhesus monkey. Exp. Neurol. 119: 220-234, 1993.

Pfingst, B. E. AND O'Connor, T. A. A vertical stereotaxic approach to auditory cortex in the unanesthetized monkey. J. Neurosci. Methods 2: 33-45, 1980.

Pfingst B. E. AND O'Connor, T. A. Characteristics of neurons in auditory cortex of monkeys performing a simple auditory task. J. Neurophysiol. 45: 16-34, 1981.

Pfingst, B. E., O'Connor, T. A., And Miller, J. M. Response plasticity of neurons in auditory cortex of the rhesus monkey. Exp. Brain Res. 29: 393-404, 1977

RAUSCHECKER, J. P. Parallel processing in the auditory cortex of primates. Audiol. Neuro-Otol. 3: 86-103, 1998.

Rauschecker, J. P., Tian, B., and Hauser, M. Processing of complex sounds in the macaque nonprimary auditory cortex. Science 268: 111-114, 1995.

Rauschecker, J. P, Tian, B., Pons, T., and Mishinin, M. Serial and parallel processing in rhesus monkey auditory cortex. J. Comp. Neurol. 382: 89103, 1997.

Recanzone, G. H., Guard, D. C., Phan, M. L., and Su, T. K. Correlation between the activity of single auditory cortical neurons and sound localization behavior in the macaque monkey. J. Neurophysiol. In press.

Recanzone, G. H., Schreiner, C. E., Sutter, M. L., Beitel, R. E., AND Merzenich, M. M. Functional organization of spectral receptive fields in the primary auditory cortex of the owl monkey. J. Comp. Neurol. 415: 460481, 1999.

Ryan, A. F., Miller, J. M., Pfingst, B. E., and Martin, G. K. Effects of reaction time performance on single unit activity in the central auditory pathways of the rhesus macaque. J. Neurosci. 4: 298-308, 1984.

SCHREINER, C. E. Spatial distribution of responses to simple and complex sounds in the primary auditory cortex. Audiol Neuro-Otol. 3: 104-122, 1998.

SChreiner, C. E. AND MEndelson, J. R. Functional topography of cat primary auditory cortex: distribution of integrated excitation. J Neurophysiol. 64: 1442-1459, 1990.

Schreiner, C. E., Mendelson, J. R., And Sutter, M. L. Functional topography of cat primary auditory cortex: representation of tone intensity. Exp Brain Res. 92: 105-122, 1992.

SCHREINER, C. E. AND SutTer, M. L. Topography of excitatory bandwidth in cat primary auditory cortex: single-neuron versus multiple-neuron recordings. J Neurophysiol. 68: 1487-1502, 1992.

Stebbins, W. C., Green, S., and Miller, F. L. Auditory sensitivity of the monkey. Science 153: 1646-1647, 1966.

SutTer, M. L. AND SCHReiner, C. E. Topography of intensity tuning in cat primary auditory cortex: single-neuron versus multiple-neuron recordings. J. Neurophysiol. 73: 190-204, 1995.

Ungerleider, L. G. AND HaXBY, J. V. "What" and "where" in the human brain. Curr. Opin. Neurobiol. 4: 157-165, 1994.

UngerLeider, L. G. AND Mishrin, M. Two cortical visual systems. In: Analysis of Visual Behavior, edited by D. J. Ingle, M. A. Goodale, and J. W. Mansfield. Cambridge, MA: MIT Press, 1982, p. 549-556. 